\title{
Changes in anthropometric and blood 25-hydroxyvitamin D measurements in antenatal vitamin supplemented gestational diabetes mellitus patients: a systematic review and meta-analysis of randomized controlled trials
}

\author{
(D) Sumanta Saha ${ }^{1}$, (D) Sujata Saha ${ }^{2}$ \\ 1R. G. Kar Medical College, Kolkata, India \\ 2Mankar College, Mankar, India
}

\section{Abstract}

Objective: Gestation weight (GW), body mass index (BMI), and blood 25-hydroxyvitamin D [25(OH)D] level during pregnancy are important determinants of the gestational outcomes. This study aimed to study how these parameters vary between antenatal vitamin $\mathrm{D}$ recipients and non-recipients in gestational diabetes mellitus (GDM) patients.

Material and Methods: The randomized controlled trials comparing these outcomes between vitamin D recipient and non-recipient GDM patients were searched in electronic databases (PubMed, Embase, and Scopus). The reviewed studies' data were abstracted and critically appraised using the Cochrane tool. The estimation of the weighted mean difference for GW and BMI and standardized mean difference (SMD) for $25(\mathrm{OH}) \mathrm{D}$ levels occurred by juxtaposing the interventions meta-analytically (random-effect model). The statistical inconsistency was determined by $\mathrm{Chi}^{2}$ and $\mathrm{I}^{2}$ method. The statistical significance was estimated at $\mathrm{p}<0.05$ and $95 \%$ confidence interval (CI).

Results: Eleven eligible trials (all Iran-based, except one), sourcing data from about 875 GDM patients, were reviewed. Overall, the risk of bias was low, except for selection and performance bias. On random-effect model meta-analysis, the 25(OH)D levels of the GDM patients favored the vitamin D recipients when compared to non-vitamin D (SMD 1.97, 95\% CI: 1.06-2.88, p <0.001; I ${ }^{2}$ 96.2\%, p of Chi ${ }^{2}<0.001$ ) and placebo (SMD 1.86, 95\% CI: $0.95-2.77, \mathrm{p}<0.001 ; \mathrm{I}^{2} 95.3 \%$, p of $\mathrm{Chi}^{2}<0.001$ ) recipients, respectively. On meta-regression, sample size was a predictor of the observed heterogeneity. For GW and BMI the interventions did not differ statistically significantly.

Conclusion: In GDM patients, antenatal use of vitamin D aids in the rise of blood 25(OH)D levels. However, vitamin D supplementation did not affect change in GW or BMI. (J Turk Ger Gynecol Assoc 2021; 22: 217-34)

Keywords: Gestational diabetes, vitamin D, dietary supplement

Received: 18 November, 2020 Accepted: 12 January, 2021

\section{Introduction}

Gestational diabetes mellitus (GDM) refers to any degree of glucose intolerance that develops or is identified initially during gestation (1). Its global prevalence is about 7-10\% (2-5). GDM diagnosis is made using glucose challenge tests between 24-28 weeks of gestation (1). Initial GDM management encompasses diet and exercise therapy, but if these fail to achieve glycemic control, physicians start insulin therapy (1).

GDM is a crucial health burden since it can affect both the GDM patient and her neonate. Gestational weight (GW) 
and body mass index (BMI) in pregnancy are two important anthropometric determinants of GDM-related outcomes. Studies in GDM patients suggest that an excessive GW accumulation increases the risk of maternal complications, such as increased likelihood of cesarean delivery, large for gestational age, and gestational hypertension and fetal problems, such as macrosomia, large for gestational age, hypoglycemia in newborns, and poor APGAR score (6-11). It remains unclear if the Institute of Medicine's guideline (2009) for recommended GW gain for respective BMI categories can be applied to the GDM subpopulation or not $(8,12)$. However, studies on overweight and obese GDM patients found that gaining GW less than that recommended for their respective BMI categories resulted in favorable obstetric and neonatal outcomes $(8,13,14)$. Maintaining an optimum weight before and during pregnancy, therefore, can decrease the complications of pregnancy (9). Nevertheless, it remains unclear if any antenatal intervention in GDM patients may be beneficial in achieving an acceptable GW and BMI.

In this respect, vitamin D has emerged as a potentially useful agent that has attracted attention. In GDM patients, various clinical trials (15-18) have tested the maternal health effect of antenatal vitamin D supplementation, and due to the different relationships between GDM and vitamin D status in the body, such testing appears pertinent. For instance, inadequate vitamin D levels in the body are associated with an increased risk of developing GDM (19-23). Vitamin D deficiency $(<20 \mathrm{ng} / \mathrm{mL})$ has a nearly fourfold increased risk of GDM development than women with sufficient vitamin D level (>30 ng/mL) after adjusting for the age of the mother, race, ethnicity, and family history of type 2 diabetes among first-degree family members (24). Moreover, studies showed a decreased GDM prevalence in prenatal vitamin D recipients $(25,26)$. Given this evidence it is important to understand how vitamin D supplementation in GDM mothers can affect GW and their BMI. Additionally, as the fetus entirely depends on maternal 25-hydroxyvitamin D [25(OH)D] levels, maternal levels in GDM mothers after vitamin D supplementation also requires evaluation (27).

\section{Intervention description}

The inactive forms of the fat-soluble vitamin D are D2 (ergocalciferol) and D3 (cholecalciferol) $(28,29)$. Both forms are available from diet and supplements and vitamin D3 is also produced in the skin on exposure to sunlight $(28,29)$. On hydroxylation of pre-vitamin $\mathrm{D}$ in the liver, the main circulating form, 25(OH)D, of vitamin D is produced (27). In blood, 25(OH) $\mathrm{D}$ is either present in the bound form (to albumin) or free form (27). For its physiologic role, it is converted to the active form, calcitriol 1,25-dihydroxyvitamin D $(28,30)$. The physiological effect of Vitamin D in pregnancy is mediated via calcitriol's action on the vitamin $\mathrm{D}$ receptors in uteroplacental tissue $(28,30)$. Compared to calcitriol, which has a half-life of 4-6 hours (27), the relatively longer half-life of 25(OH)D of between two and three weeks (31) makes the latter an ideal marker for vitamin D status (32).

In GDM patients, contemporary trials have supplemented vitamin D at various dosages. For oral preparations, while some trials used it at a dose of 50,000 IU, two to three weeks apart for three to eight weeks (33-36), other trials used it twice daily at 200-500 IU for six to sixteen weeks $(17,37)$. One trial used a single intramuscular injection of vitamin D at a dose of 300,000 IU (38). Furthermore, while few trials used the vitamin as a single supplement $(33,37,38)$, others co-supplemented it with various micronutrients, including zinc, magnesium, and calcium $(17,34)$.

\section{What this review adds?}

In contemporary medicine, several clinical trials have tested the changes in GW, BMI, and plasma 25(OH)D level in GDM mothers, after antenatal vitamin D supplementation (15,34-36). Recent reviews have studied the effect of antenatal vitamin D supplementation on certain maternal complications such as cesarean section rate, pre-eclampsia, preterm delivery, macrosomia, and polyhydramnios and/or on neonatal complications including hyperbilirubinemia, hypoglycemia, and hospitalization (39-41). However, to the best of our knowledge, there is no systematic review and meta-analysis that studied how maternal GW, BMI, and 25(OH)D levels change in the blood on vitamin D supplementation in GDM patients. Therefore, this study explores this under-reviewed area of modern medicine by a systematic literature search, critical appraisal, and meta-analysis.

\section{Aims}

This study compared the GW, BMI, and 25(OH)D levels among vitamin D supplemented and not-supplemented GDM patients.

\section{Material and Methods}

This systematic review is registered in the PROSPERO database (CRD42020149613) and has a pre-published protocol $(42,43)$. This report adheres to the PRISMA 2009 reporting guideline (Supplement Table 1) (44).

\section{Inclusion criteria}

1. Study design: Parallel arm randomized controlled trials of any number of intervention arms.

2. Population: Pregnant females of any age were eligible, irrespective of their pre-pregnancy BMI and 25(OH)D levels. 
They must be diagnosed with GDM during their concurrent pregnancy.

3. Intervention arm: The treatment arm/s should have received vitamin D as a sole or co-supplement.

4. Comparator arm: The comparator arm/s may have received a placebo or any other supplement except vitamin D. Comparator arm/s not receiving any intervention were also eligible.

5. Outcomes: The trials must report the GW $(\mathrm{kg})$, BMI $\left(\mathrm{kg} / \mathrm{m}^{2}\right)$, and $25(\mathrm{OH}) \mathrm{D}$ (in $\mathrm{ng} / \mathrm{mL}$ or $\mathrm{mmol} / \mathrm{L}$ ) in the above GDM patients before and after receiving these interventions and before childbirth.

We accepted the diagnosis and management of GDM and the dosage and regimen of interventions received by the participants in the respective treatment arms as per the trialists.

\section{Exclusion criteria}

1. Study design other than those described above, e.g., observational studies and crossover studies.

2. Participants with diabetes types besides GDM, like type 1 or type 2 diabetes.

3. Studies conducted on animals.

4. Editorials, abstracts from conference presentations (where a full published manuscript is not available), letters, or any other brief communications.

\section{Database search}

We searched the title and abstract of prospective trials matching the above eligibility criteria in PubMed, Embase, and Scopus databases, irrespective of the date and language of publication and geographical boundary. The following search terms were used "vitamin D" OR "calciferol" OR "vitamin D2" OR "ergocalciferol" OR "vitamin D3" OR "cholecalciferol" AND "GDM" OR "gestational diabetes" along with these MeSH terms- "Cholecalciferol", "ergocalciferols", and "diabetes, gestational". To identify the clinical trials in PubMed [(Clinical Trial) and (Randomized Controlled Trial)] and Embase [(controlled clinical trial) and (randomized controlled trial)], we used filters. In Scopus, instead of filters, the following search terms were used: "trial," "randomised," "randomized," and "controlled." The last date of the search was 17 September, 2020. Additionally, we reviewed the references of the papers included in this review.

We uploaded the retrieved citations (from database search) in the Rayyan systematic review software (45) and eliminated the duplicate articles. Successively, skimming of the remaining citations' titles and abstracts against the eligibility criteria commenced. Articles were read in full-text when it seemed to meet the inclusion criteria, or if the suitability for incorporation in this review was doubtful.

\section{Data abstraction and risk of bias assessment}

We extracted data about the study design, consent, ethics, registration number of the trial, participant features, interventions contrasted, and the outcomes of interest in a prepiloted form. With the Cochrane collaboration tool, individual trial's risk of selection bias, performance bias, detection bias, attrition bias, reporting bias, and any other bias was determined, and each of these risk of bias (RoB) components was categorized as low, high, or unclear (46). To assess selection bias, the random allocation sequence generation method, and its concealment method from participants, were judged. The blinding mechanism of study participants and personnel and that of outcome assessors were used to evaluate the performance and detection bias, respectively. By evaluating missing outcome data, and its reason among the intervention arms, the risk of attrition bias, was evaluated. Any additional bias, besides the above, comprised the other bias type. For a visual presentation of the RoB, we prepared an RoB graph and an RoB summary using the Review Manager (RevMan) software $(46,47)$.

The review authors independently performed study selection, data abstraction, and RoB assessment, and resolved any disagreement in an opinion by discourse.

\section{Meta-analysis}

The juxtaposed interventions' effect on each of the outcomes was contrasted by random-effects meta-analysis (using DerSimonian and Laird method) since we assumed clinical heterogeneity among the trials attributable to the different types of vitamin D co-supplements used in these. The use of endpoint means of the respective outcomes and their SDs ensued to conduct the meta-analysis. We estimated the meta-analytic effect sizes of GW and BMI in weighted mean differences (WMD) and that of 25(OH)D levels in standardized mean differences (SMD) due to the identical and non-identical types of measuring units used in the trials, respectively. A decrease in the summary effect of GW and BMI, and its increase in 25(OH)D levels, denoted a favorable meta-analytic finding. For any outcome, when multiple treatment arms tested an intervention, the post-intervention means and their SDs of those intervention groups were combined for meta-analysis (46). Outcome reported in the median were not considered for meta-analysis.

\section{Heterogeneity and meta-regression}

The statistical heterogeneity was determined by $\mathrm{Chi}^{2}$ (statistically significant at $\mathrm{p}<0.1$ ) and $\mathrm{I}^{2}$ (categorized as low, 
moderate, and high at $\mathrm{I}^{2}$ values of 25,50 , and $75 \%$, respectively) statistics (48). To account for any substantial heterogeneity, we performed univariate meta-regression by presence or absence of missing outcome data and sample size (categorized as $<100$ and $\geq 100$ ). Using the predictor identified by meta-regression, we did a subgroup analysis to see how heterogeneity changed across the different categories of the predictor.

\section{Publication bias and sensitivity analysis}

The publication bias assessment incorporated visual inspection of funnel plots and Egger's test. For each outcome, a sensitivity analysis included iteration of the meta-analysis using a fixedeffect model and by dropping a trial each time.

\section{Statistical analysis}

Using random-effect and fixed-effect models, all outcomes were compared meta-analytically between vitamin $\mathrm{D}$ and placebo-receiving GDM patients.

We estimated the statistical significance of meta-analysis derived effect sizes at $\mathrm{p}<0.05$ and $95 \%$ confidence interval (CI). Stata statistical software v16 (StataCorp, College Station, TX) was used for analysis.

\section{Results}

\section{Scope of the review}

The database search retrieved 271 citations. After eliminating the duplicates, 188 citations underwent skimming against the eligibility criteria. Out of the 22 articles needing full-text reading, 11 trials sourcing data from about 875 participants published between 2014-19, were included in this review (Figure 1) (15-18,33-35,37,49-51). All trials except the Chinese one (37) were Iran-based, and the average age of participants in the respective intervention arms was approximately 28-32 years. The intervention period of Iranian (16-18,33,49-51) and Chinese (37) trials were 6-8 and 16 weeks, respectively. In most trials (15-18,34,35,37,49-51), GDM was diagnosed primarily using the American Diabetes Association criteria $(52,53)$. Insulin was not used during the intervention period, except in the trial by Yazdchi et al. (33). Eight trials $(15,16,18,33,34,37,49,50)$ used the D3 form of the vitamin while this was not clear among the remaining trials $(17,35,51)$. In most of the trials $(81.8 \%)$, a co-supplement (e.g., calcium, magnesium, zinc, omega-3 fatty acid, evening primrose oil, probiotic) accompanied the vitamin D supplementation (15-18,34,35,37,50,51). The intervention was given between 24-28 weeks of gestation in nine trials $(15-18,33-35,49,50)$, at 16 weeks of gestation in one trial (37), and in the remaining one, this was unclear (51). Table 1 depicts the salient features of the trials.

\section{RoB assessment}

In most studies, the allocation concealment component of the selection bias and performance bias was unclear (Table 2 and Figure 2). Otherwise, the RoB was low.

\section{Meta-analysis findings}

Eleven trials comparing GW (15,16,51,17,18,33-35,37,49,50), and 10 trials contrasting BMI (15-18,33-35,49-51) with one study (37) excluded as it did not report the follow up BMI, and $25(\mathrm{OH})$ D with one trial (33) excluded for reporting follow up value in median, were included in the meta-analytic juxtaposition between vitamin $\mathrm{D}$ recipients and its non-recipients.

The antenatal vitamin D use in GDM patients favored plasma 25(OH)D level attainment compared to its non-supplementation (random-effect model: SMD 1.97, 95\% CI: 1.06-2.88, p <0.001; I ${ }^{2}$ $96.2 \%$, p of $\mathrm{Chi}^{2}<0.001$ ).

The post-intervention GW (random-effect model: WMD 0.18, 95\% CI, -1.10-1.47, $\mathrm{p}=0.773 ; \mathrm{I}^{2}$ 0\%, $\mathrm{p}$ of $\mathrm{Chi}^{2}$ 0.559) and BMI (random-effect model: WMD 0.27, 95\% CI, -0.28-0.82, $\mathrm{p}=0.331$; $\mathrm{I}^{2} \mathrm{0} \%$, p of $\mathrm{Chi}^{2} 0.838$ ) were not statistically significantly different between the juxtaposed interventions (Figure 3).

\section{Meta-regression and subgroup analysis}

The univariate meta-regression suggested that sample size was a statistically significant predictor of the observed heterogeneity in the effect size of 25(OH)D level (Supplement Table 2). Upon subgroup analysis by the sample size, heterogeneity was moderate when sample size was $\geq 100$, and the effect size increased (random-effect model: SMD 3.81, p<0.001; 95\% CI, 3.03-4.59; I $I^{2} 72.5 \%$ ) (Supplement Figure 1).

\section{Publications bias}

For 25(OH)D, a small study effect was suggested by the asymmetric funnel plots (Supplement Figure 2) and Egger's test $(\mathrm{p}=0.005)$. On trim-and-fill analysis, no additional study was imputed. Funnel plots for the rest of the outcomes were approximately symmetric.

\section{Sensitivity analysis}

On using a fixed-effect model meta-analysis, the summary estimate of 25(OH)D level, reduced slightly (SMD 1.74, 95\% CI, 1.57-1.92, $\mathrm{p}<0.001)$. The fixed-effect meta-analysis results for the rest of the outcomes were identical to the preliminary analysis. The meta-analysis findings for all outcomes remained unchanged on dropping a study each time and repeating the meta-analysis.

\section{Supplementary meta-analysis}

Between vitamin D and placebo, ten trials (15-18,33-35,4951) compared GW and BMI, and nine trials (15-18,34,35,49- 
51) juxtaposed 25(OH)D levels, with one study (33) excluded because the study reported $25(\mathrm{OH}) \mathrm{D}$ values as medians. Vitamin D recipients achieved a favorable blood 25(OH) D level compared to the placebo recipients (random-effect model: SMD 1.86, 95\% CI, 0.95-2.77, p<0.001; I2, 95.36\%, p of $\mathrm{Chi}^{2}<0.001$ ) (Figure 4). The effect size of 25(OH)D levels reduced slightly on using a fixed-effect meta-analysis model (SMD 1.45, 95\% CI, 1.25-1.64). GW and BMI, when contrasted among the intervention arms, were not statistically significantly different. Since $<10$ studies were available for the 25(OH)D levels, we did not explore heterogeneity or assess the publication bias for it.

\section{Discussion}

Overall, 11 trials, mostly Iranian, tested the effect of antenatal vitamin $\mathrm{D}$ complementation (as a co-supplement primarily) on GW, BMI, and 25(OH)D in 875 GDM patients, were retrieved. The intervention favored a rise in blood 25(OH)D levels, and the sample size was the plausible predictor of the observed heterogeneity.

\section{Evidence quality}

Utilizing the GRADE Working Group's (2004) (54) approach of grading evidence quality we graded the evidence concerning the 25(OH)D level as of moderate-quality, due to the unclear RoB components and heterogeneity.

\section{Comparison with what is known}

As the context remains underexplored in contemporary literature, a direct juxtaposition of our findings to existing reviews is not possible. However, clinical trials studying the effect of vitamin D supplementation on 25(OH)D level in pregnant females with no glucose intolerance are available for a contrast. Two such trials found that vitamin D supplementation in the third trimester increased maternal plasma 25(OH) D levels compared to the control group $(55,56)$. Another randomized trial found that vitamin $\mathrm{D}$ supplementation caused a statistically significantly greater increase in the $25(\mathrm{OH}) \mathrm{D}$ level than the placebo (57). Mirroring these trials' findings (55-57), we observed that vitamin D3 supplementation in GDM patients increased the maternal 25(OH)D level.

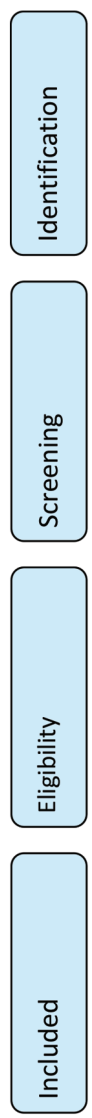

Records identified through database searching $(n=296)$ through other sources $(n=0)$

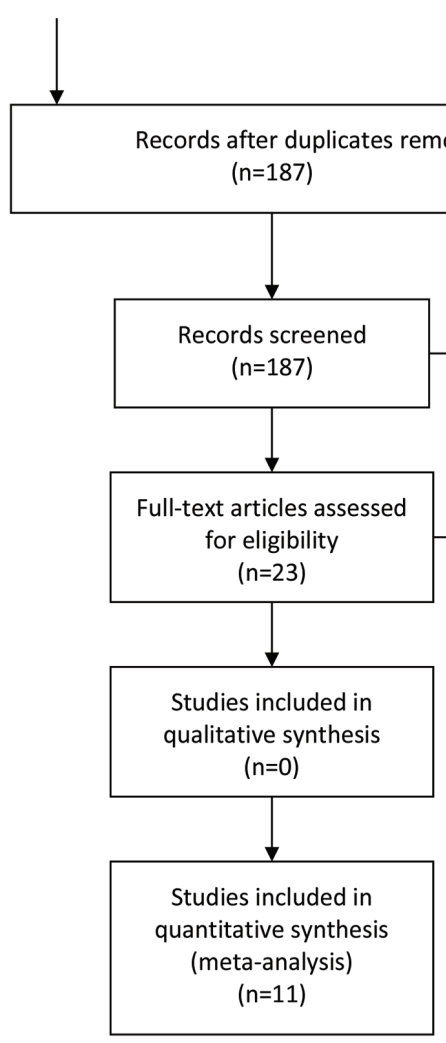

Records excluded $(n=164)$

Figure 1. Study selection process [PRISMA flow chart (58)] 
Table 1. Summary table

\begin{tabular}{|c|c|c|c|c|}
\hline Trial & Design & Population & Intervention arms & $\begin{array}{l}\text { Outcomes } \\
\text { reported }\end{array}$ \\
\hline $\begin{array}{l}\text { Karamali } \\
\text { et al. (16) }\end{array}$ & $\begin{array}{l}\text { Randomized, placebo-controlled } \\
\text { trial } \\
\text { Blinding: double blinded } \\
\text { Number of intervention arms: two } \\
\text { Multi-center or single-center trial: } \\
\text { multi-centric } \\
\text { Study duration: six weeks } \\
\text { Country where trial was } \\
\text { conducted: Iran } \\
\text { Ethical permission: obtained } \\
\text { Consent from participants: } \\
\text { obtained } \\
\text { Information regarding funding: } \\
\text { provided } \\
\text { Clinical trial registration number: } \\
\text { IRCT201407115623N23 }\end{array}$ & $\begin{array}{l}\text { Diagnosis: GDM (using ADA criteria) } \\
\text { Number of participants randomized: } 60 \\
\text { Calcium and vitamin D arm (n): } 30 \\
\text { Placebo group (n): } 30 \\
\text { Average age of Calcium and vitamin D arm: } 28.7(6.1) \\
\text { years } \\
\text { Average age of placebo group: } 31.6 \text { (6.3) years } \\
\text { Missing outcome data: } 0 \\
\text { Baseline mean BMI (SD): Placebo group: } 30.5(4.5) \mathrm{kg} / \\
\mathrm{m}^{2} \text {; Calcium and vitamin D arm: } 29.4(4.7) \mathrm{kg} / \mathrm{m}^{2} \\
\text { Baseline mean GW (SD): Placebo group: } 78.1(13.4) \mathrm{kg} \text {; } \\
\text { Calcium and vitamin D arm: } 73.7 \text { (12.8) } \mathrm{kg} \\
\text { Baseline mean (SD) vitamin D levels: Placebo group: } \\
20.8(14.4) \mathrm{ng} / \mathrm{mL} \text {; Calcium and vitamin D arm: } 17.3 \\
(10.9) \mathrm{ng} / \mathrm{mL}\end{array}$ & $\begin{array}{l}\text { Calcium and vitamin D } \\
\text { arm: calcium carbonate } \\
1000 \mathrm{mg} / \text { day (six weeks) } \\
\text { and } 50,000 \mathrm{IU} \text { D3 (at trial } \\
\text { initiation and } 21^{\text {st }} \text { day). } \\
\text { Placebo arm. } \\
\text { Intervention given } \\
\text { between } 24 \text { and } 28 \\
\text { weeks of pregnancy. } \\
\text { Total vitamin D received } \\
\text { in six weeks: } 100,000 \mathrm{IU} .\end{array}$ & $\begin{array}{l}\text { 1. GW } \\
\text { 2. BMI } \\
\text { 3. } 25(\mathrm{OH}) \mathrm{D}\end{array}$ \\
\hline $\begin{array}{l}\text { Karamali } \\
\text { et al. (17) }\end{array}$ & $\begin{array}{l}\text { Randomizedplacebo-controlled } \\
\text { trial } \\
\text { Blinding: double blinded } \\
\text { Number of intervention arms: two } \\
\text { Multi-center or single-center trial: } \\
\text { single-centric } \\
\text { Study duration: six weeks } \\
\text { Country where trial was } \\
\text { conducted: Iran } \\
\text { Ethical permission: obtained } \\
\text { Consent from participants: not } \\
\text { clear } \\
\text { Information regarding funding: } \\
\text { provided } \\
\text { Clinical trial registration number: } \\
\text { not available }\end{array}$ & $\begin{array}{l}\text { Diagnosis: GDM (using ADA criteria) } \\
\text { Number of participants randomized: } 60 \\
\text { Magnesium, zinc, calcium and vitamin D supplements } \\
\text { arm (n): } 30 \\
\text { Placebo group (n): } 30 \\
\text { Average age of magnesium, zinc, calcium and vitamin D } \\
\text { supplements arm: } 30 \text { (4.5) years } \\
\text { Average age of placebo group: } 31.1 \text { (4.2) years } \\
\text { Missing outcome data: } 0 \\
\text { Baseline mean BMI (SD): Placebo group: } 27(2.6) \mathrm{kg} / \mathrm{m}^{2} \text {; } \\
\text { Magnesium, zinc, calcium and vitamin D supplements } \\
\text { arm: } 27.4 \text { (4.8) } \mathrm{kg} / \mathrm{m}^{2} \\
\text { Baseline mean GW (SD): Placebo group: } 70.7(7.2) \mathrm{kg} \text {; } \\
\text { Magnesium, zinc, calcium and vitamin D supplements } \\
\text { arm: } 70.9 \text { (12.8) } \mathrm{kg} \\
\text { Baseline mean (SD) vitamin D levels: Placebo group: } \\
20.21 \text { (10.73) ng/mL; } \\
\text { Magnesium, zinc, calcium and vitamin D supplements } \\
\text { arm: } 18.96 \text { (11.23) ng/mL }\end{array}$ & $\begin{array}{l}\text { Magnesium, calcium, } \\
\text { zinc and vitamin D arm: } \\
100 \text { mg magnesium, } 400 \\
\text { mg calcium, } 4 \text { mg zinc, } \\
\text { and } 200 \mathrm{IU} \text { vitamin D } \\
2 \mathrm{x} / \mathrm{d} \text { (six weeks). } \\
\text { Placebo arm. } \\
\text { Total vitamin D received } \\
\text { in six weeks: } 16,800 \mathrm{IU}\end{array}$ & $\begin{array}{l}\text { 1. GW } \\
\text { 2. BMI } \\
\text { 3. } 25(\mathrm{OH}) \mathrm{D}\end{array}$ \\
\hline $\begin{array}{l}\text { Asemi et } \\
\text { al. (49) }\end{array}$ & $\begin{array}{l}\text { Randomized, placebo-controlled } \\
\text { trial } \\
\text { Blinding: double blinded } \\
\text { Number of intervention arms: two } \\
\text { Multi-center or single-center trial: } \\
\text { multi-centric } \\
\text { Study duration: six weeks } \\
\text { Country where trial was } \\
\text { conducted: Iran } \\
\text { Ethical permission: obtained } \\
\text { Consent from participants: } \\
\text { obtained } \\
\text { Information regarding funding: } \\
\text { provided } \\
\text { Clinical trial registration number: } \\
\text { IRCT201305115623N7 }\end{array}$ & $\begin{array}{l}\text { Diagnosis: GDM (using ADA criteria) } \\
\text { Number of participants randomized: } 50 \\
\text { Vitamin D arm (n): } 25 \\
\text { Placebo group (n): } 25 \\
\text { Average age of vitamin D arm: } 31.1 \text { (5.5) years } \\
\text { Average age of placebo group: } 30.8 \text { (6.2) years } \\
\text { Missing outcome data: } 5 \text { (three in vitamin D armand two } \\
\text { in placebo arm); Causes of missingness: intra-uterine } \\
\text { fetal death (n=1), placenta abruption ( } \mathrm{n}=1 \text { ), completed } \\
\text { bed rest (n=1), insulin therapy (n=1), pre-eclampsia } \\
\text { (n=1) } \\
\text { Baseline mean BMI (SD): Placebo group: } 30.5(4.5) \mathrm{kg} / \\
\mathrm{m}^{2} \text {; Vitamin D arm: } 30.7 \text { (3.9) kg/m² } \\
\text { Baseline mean GW (SD): Placebo group: } 77.8(12.9) \mathrm{kg} \text {; } \\
\text { Vitamin D arm: } 79.0 \text { (9.7) kg } \\
\text { Baseline mean (SD) vitamin D levels: Placebo group: } \\
20.9 \text { (14.3) ng/mL; Vitamin D arm: } 18.9 \text { (14.5) ng/mL }\end{array}$ & $\begin{array}{l}\text { Vitamin D arm: } 50,000 \mathrm{IU} \\
\text { D3 (at trial initiation and } \\
21^{\text {st }} \text { day). } \\
\text { Placebo arm. } \\
\text { Total vitamin D received } \\
\text { in six weeks: } 100,000 \mathrm{IU} .\end{array}$ & $\begin{array}{l}\text { 1. GW } \\
\text { 2. BMI } \\
\text { 3. } 25(\mathrm{OH}) \mathrm{D}\end{array}$ \\
\hline
\end{tabular}


Table 1. Continued

\begin{tabular}{|c|c|c|c|c|}
\hline Trial & Design & Population & Intervention arms & $\begin{array}{l}\text { Outcomes } \\
\text { reported }\end{array}$ \\
\hline $\begin{array}{l}\text { Jamilian } \\
\text { et al. (50) }\end{array}$ & $\begin{array}{l}\text { Randomized, placebo-controlled } \\
\text { trial } \\
\text { Blinding: double blinded } \\
\text { No. of treatment arms: two } \\
\text { Single centered trial } \\
\text { Study duration: six weeks } \\
\text { Country where trial was } \\
\text { conducted: Iran } \\
\text { Ethical permission: obtained } \\
\text { Consent from participants: } \\
\text { obtained } \\
\text { Information regarding } \\
\text { fundingInformation regarding } \\
\text { funding: provided } \\
\text { Clinical trial registration number: } \\
\text { IRCT201706075623N119 }\end{array}$ & $\begin{array}{l}\text { Diagnosis: GDM (using ADA criteria) } \\
\text { Number of participants randomized: } 90 \\
\text { Probiotic and vitamin D arm (n): } 30 \\
\text { Probiotic arm (n): } 30 \\
\text { Placebo group (n): } 30 \\
\text { Average age of probiotic and vitamin D arm: } 28.9 \\
\text { (6.1) years } \\
\text { Average age of probiotic group: } 31.2 \text { (5.9) years } \\
\text { Average age of placebo group: } 29.9 \text { (3.7) years } \\
\text { Missing outcome data: } 3 \text {; Causes of missingness: } \\
\text { insulin therapy (n=1) and hospitalization (n=1) } \\
\text { Baseline mean BMI (SD): Placebo group: } 27.5(3.3) \\
\text { kg/m²; Probiotic and vitamin D arm: } 27.8(4.9) \mathrm{kg} / \mathrm{m}^{2} \text {; } \\
\text { Probiotic group: } 26.4 \text { (4.2) kg/m² } \\
\text { Baseline mean GW (SD): Placebo group: } 72.0(7.7) \\
\text { kg; Probiotic and vitamin D arm: } 71.9 \text { (12.1) kg; } \\
\text { Probiotic group: } 70.0 \text { (12.5) kg } \\
\text { Baseline mean (SD) vitamin D levels: Placebo } \\
\text { group: } 14.3 \text { (4.1) ng/mL; Probiotic and vitamin D } \\
\text { arm: } 13.4 \text { (4.1) ng/mL; Probiotic group: } 12.9(3.2 \mathrm{ng} / \\
\text { mL) }\end{array}$ & $\begin{array}{l}\text { Probiotic and vitamin D } \\
\text { arm: } 50,000 \text { IU D3 (every } \\
2 \text { weeks) and } 8 * 10^{9} \\
\text { CFU/g probiotic } \\
\text { Probiotic arm: } 8^{*} 10^{9} \\
\text { CFU/g probiotic } \\
\text { Placebo arm. } \\
\text { Total vitamin D received } \\
\text { in six weeks: } 150,000 \mathrm{IU}\end{array}$ & $\begin{array}{l}\text { 1. } \mathrm{GW} \\
\text { 2. } \mathrm{BMI} \\
\text { 3. } 25(\mathrm{OH}) \mathrm{D}\end{array}$ \\
\hline $\begin{array}{l}\text { Jamilian } \\
\text { et al. (18) }\end{array}$ & $\begin{array}{l}\text { Randomized, placebo-controlled } \\
\text { trial } \\
\text { Blinding: double blinded } \\
\text { No. of treatment arms: two } \\
\text { Single centered trial } \\
\text { Study duration: six weeks } \\
\text { Country where trial was } \\
\text { conducted: Iran } \\
\text { Ethical permission: obtained } \\
\text { Consent from participants: } \\
\text { obtained } \\
\text { Information regarding funding: } \\
\text { provided } \\
\text { Clinical trial registration number: } \\
\text { IRCT201704225623N109 }\end{array}$ & $\begin{array}{l}\text { Diagnosis: GDM (using ADA criteria) } \\
\text { Number of participants randomized: } 60 \\
\text { Magnesium, zinc, calcium plus vitamin D arm (n): } 30 \\
\text { Placebo group (n): } 30 \\
\text { Average age of magnesium, zinc, calcium plus } \\
\text { vitamin D arm: } 27.7 \text { (4.0) years } \\
\text { Average age of placebo group: } 29.1 \text { (4.1) years } \\
\text { Missing outcome data: 0 } \\
\text { Baseline mean BMI (SD): Placebo group: } 25.3 \text { (2.5) } \\
\text { kg/m²; magnesium, zinc, calcium plus vitamin D } \\
\text { arm: } 25.8 \text { (3.7) kg/m² } \\
\text { Baseline mean GW (SD): Placebo group: } 67.6 \text { (6.1) } \\
\text { kg; Magnesium, zinc, calcium plus vitamin D arm: } \\
68.2 \text { (9.4) kg } \\
\text { Baseline mean (SD) vitamin D levels: Placebo } \\
\text { group: } 13.5 \pm 3.6 \mathrm{ng} / \mathrm{mL} ; \text { Magnesium, zinc, calcium } \\
\text { plus vitamin D arm: } 12.6 \pm 4.2 \text { ng/mL }\end{array}$ & $\begin{array}{l}\text { Magnesium, calcium, } \\
\text { zinc, and vitamin D arm: } \\
100 \mathrm{mg} \text { magnesium, } \\
400 \mathrm{mg} \text { calcium, } 4 \mathrm{mg} \\
\text { zinc, and } 200 \mathrm{IU} \text { D3: } \\
\text { two times daily for six } \\
\text { weeks. } \\
\text { Placebo arm. } \\
\text { Total vitamin D received } \\
\text { in six weeks: } 16800 \mathrm{IU}\end{array}$ & $\begin{array}{l}\text { 1. GW } \\
\text { 2. BMI } \\
\text { 3. } 25(\mathrm{OH}) \mathrm{D}\end{array}$ \\
\hline $\begin{array}{l}\text { Li and } \\
\text { Xing (37) }\end{array}$ & $\begin{array}{l}\text { Randomized, clinical trial } \\
\text { Blinding: double blinded } \\
\text { No of treatment arms: two } \\
\text { Multricentric trial } \\
\text { Study duration: } 16 \text { weeks } \\
\text { Country where trial was } \\
\text { conducted: China } \\
\text { Ethical permission: obtained } \\
\text { Consent from participants: } \\
\text { obtained } \\
\text { Information regarding funding: not } \\
\text { clear } \\
\text { Clinical trial registration number: } \\
\text { not clear }\end{array}$ & $\begin{array}{l}\text { Diagnosis: GDM (using ADA criteria) } \\
\text { Number of participants randomized: } 103 \\
\text { Yoghurt supplemented with vitamin D arm (n): } 52 \\
\text { Plain yoghurt group (n): } 51 \\
\text { Average age of yoghurt supplemented with vitamin } \\
\text { D arm: } 29.0 \pm 5.3 \text { years } \\
\text { Average age of plain yoghurt group: } 28.3 \pm 4.1 \text { years } \\
\text { Missing outcome data: } 6 \text { [non-compliance (3) and } \\
\text { personal reasons (3)] } \\
\text { Baseline mean GW (SD): Plain yoghurt group } \\
69.3 \pm 6.7 \text { kg; Yoghurt supplemented with vitamin D } \\
\text { arm: } 67.9 \pm 7.1 \text { ) kg } \\
\text { Baseline mean (SD) vitamin D levels: Plain yoghurt } \\
\text { group: } 16.2(3.4) \mathrm{ng} / \mathrm{mL} ; \text { Yoghurt supplemented with } \\
\text { vitamin D arm: } 16.8 \pm 4.6 \text { ) ng/mL }\end{array}$ & $\begin{array}{l}\text { Yoghurt and vitamin D } \\
\text { arm: plain yoghurt and } \\
500 \text { IU D3 (twice daily } \\
\text { for } 16 \text { weeks) } \\
\text { Plain yoghurt arm: twice } \\
\text { daily for } 16 \text { weeks. } \\
\text { Total vitamin D received } \\
\text { in six weeks: } 112,000 \mathrm{IU}\end{array}$ & $\begin{array}{l}\text { 1. GW } \\
\text { 2. } 25(\mathrm{OH}) \mathrm{D} \\
\text { level }\end{array}$ \\
\hline
\end{tabular}


Table 1. Continued

\begin{tabular}{|c|c|c|c|c|}
\hline Trial & Design & Population & Intervention arms & $\begin{array}{l}\text { Outcomes } \\
\text { reported }\end{array}$ \\
\hline $\begin{array}{l}\text { Razavi et } \\
\text { al. (51) }\end{array}$ & $\begin{array}{l}\text { Randomized clinical trial } \\
\text { Blinding: double blinded } \\
\text { No. of treatment arms: two } \\
\text { Single centered trial } \\
\text { (59) } \\
\text { Study duration: six weeks. } \\
\text { Country where trial was } \\
\text { conducted: Iran } \\
\text { Ethical permission: obtained } \\
\text { Consent from participants: } \\
\text { obtained } \\
\text { Information regarding funding: } \\
\text { provided } \\
\text { Clinical trial registration number: } \\
\text { IRCT201701305623N106 }\end{array}$ & $\begin{array}{l}\text { Diagnosis: GDM (using ADA criteria) } \\
\text { Number of participants randomized: } 120 \\
\text { Vitamin D arm (n): } 30 \\
\text { Omega-3 arm (n): } 30 \\
\text { Vitamin D and Omega-3 arm (n): } 30 \\
\text { Placebo arm (n): } 30 \\
\text { Average age of Vitamin D arm: } 29.9 \pm 5.0 \text { years } \\
\text { Average age of Omega-3 arm: } 29.7 \pm 3.6 \text { years } \\
\text { Average age of vitamin D and Omega-3 arm: } 29.9 \pm 4.0 \\
\text { years } \\
\text { Average age of placebo arm: } 29.2 \pm 3.4 \text { years } \\
\text { Missing outcome data: } 0 \\
\text { Baseline mean GW (SD): Vitamin D arm: } 76.1 \pm 12.7 \mathrm{~kg} \text {; } \\
\text { Omega-3 arm: } 74.3 \pm 5.8 \mathrm{~kg} ; \text { vitamin D and Omega-3 } \\
\text { arm: } 77.4 \pm 10.2 \mathrm{~kg} ; \text { Placebo arm: } 75.1 \pm 7.7 \mathrm{~kg} \\
\text { Baseline mean (SD) BMI: Vitamin D arm: } 29.2 \pm 5.0 \\
\mathrm{~kg} / \mathrm{m}^{2} ; \text { Omega-3 arm: } 28.5 \pm 2.4 \mathrm{~kg} / \mathrm{m}^{2} ; \text { vitamin D and } \\
\text { Omega-3 arm: } 29.5 \pm 3.8 \mathrm{~kg} / \mathrm{m}^{2} ; \text { placebo arm: } 28.8 \pm 3.4 \\
\mathrm{~kg} / \mathrm{m}^{2} \\
\text { Baseline mean (SD) vitamin D levels: Vitamin D arm: } \\
13.6 \pm 3.7 \text { ng/mL; Omega-3 arm: } 15.6 \pm 4.0 \mathrm{ng} / \mathrm{mL} ; \\
\text { Vitamin D and Omega-3 arm: } 14.2 \pm 2.9 \text { ng } / \mathrm{mL} ; \text { placebo } \\
\text { arm: } 14.9 \pm 3.2 \text { ng/mL }\end{array}$ & $\begin{array}{l}\text { Vitamin D arm: } 50,000 \mathrm{IU} \\
\text { (two weekly) } \\
\text { Omega-3 arm: } 1,000 \mathrm{mg} \\
\text { omega-3 fatty acids two } \\
\text { times a day } \\
\text { Vitamin D and Omega-3 } \\
\text { arm: } 50,000 \text { IU Vitamin D } \\
\text { (two weekly) and 1,000 } \\
\text { mg omega-3 fatty acids: } \\
\text { two times a day for six } \\
\text { weeks. } \\
\text { Placebo arm. } \\
\text { Total vitamin D received } \\
\text { in six weeks: } 150,000 \mathrm{IU}\end{array}$ & $\begin{array}{l}\text { 1. GW } \\
\text { 2. BMI } \\
\text { 3. } 25(\mathrm{OH}) \mathrm{D}\end{array}$ \\
\hline $\begin{array}{l}\text { Yazdchi } \\
\text { et al. (33) }\end{array}$ & $\begin{array}{l}\text { Randomized controlled clinical } \\
\text { trial } \\
\text { Blinding: double blinded } \\
\text { No of treatment arms: two } \\
\text { Single centered trial } \\
\text { Study duration: } 8 \text { weeks. } \\
\text { Country where trial was } \\
\text { conducted: Iran } \\
\text { Ethical permission: obtained } \\
\text { Consent from participants: } \\
\text { obtained } \\
\text { Information regarding funding: } \\
\text { provided } \\
\text { Clinical trial registration number: } \\
\text { IRCT201306253140N11 }\end{array}$ & $\begin{array}{l}\text { Diagnosis: GDM (using International Association of } \\
\text { Diabetes and Pregnancy Study Groups criteria) } \\
\text { Number of participants randomized: } 76 \\
\text { Vitamin D arm (n): } 38 \\
\text { Placebo arm (n): } 38 \\
\text { Average age of Vitamin D arm: } 31.64 \pm 4.40 \text { years } \\
\text { Average age of placebo arm: } 32.11 \pm 3.61 \text { years } \\
\text { Missing outcome data: } 4 \text { [ severe preeclampsia (1), } \\
\text { early childbirth (1), unwilling to continue (1), and } \\
\text { hospitalization (1)] } \\
\text { Baseline mean GW (SD): Vitamin D arm: } 81.48 \pm 10.79 \\
\text { kg; Placebo arm: } 81.09 \pm 9.80 \mathrm{~kg} \\
\text { Baseline mean (SD) BMI: Vitamin D arm: } 31.51 \pm 3.74 \\
\text { kg/m²; placebo arm: } 31.47 \pm 3.71 \mathrm{~kg} / \mathrm{m}^{2} \\
\text { Vitamin D levels data was reported in median }\left(25^{\text {th }} \text { and }\right. \\
75^{\text {th }} \text { percentiles) due to non-parametric distribution: } \\
\text { Baseline: Vitamin D arm: } 9.54(6.12-15.94) \mathrm{ng} / \mathrm{mL} ; \\
\text { placebo arm: } 9.02 \text { ( } 7.29-14.70) \mathrm{ng} / \mathrm{mL}\end{array}$ & $\begin{array}{l}\text { Vitamin D arm: } 50,000 \text { IU } \\
\text { D3 (two weekly) } \\
\text { Placebo arm. } \\
\text { Total vitamin D received } \\
\text { in eight weeks: } 200,000 \\
\text { IU }\end{array}$ & $\begin{array}{l}\text { 1. GW } \\
\text { 2. BMI } \\
\text { 3. } 25(\mathrm{OH}) \mathrm{D}\end{array}$ \\
\hline $\begin{array}{l}\text { Asemi et } \\
\text { al. (34) }\end{array}$ & $\begin{array}{l}\text { Randomized clinical trial } \\
\text { Blinding: double blinded } \\
\text { No. of treatment arms: two } \\
\text { Multicentric trial } \\
\text { Study duration: six weeks. } \\
\text { Country where trial was } \\
\text { conducted: Iran } \\
\text { Ethical permission: obtained } \\
\text { Consent from participants: } \\
\text { obtained } \\
\text { Information regarding funding: } \\
\text { provided } \\
\text { Clinical trial registration number: } \\
\text { IRCT201311205623N11 }\end{array}$ & $\begin{array}{l}\text { Diagnosis: GDM (using ADA criteria) } \\
\text { Number of participants randomized: } 56 \\
\text { Vitamin D and calcium arm (n): } 28 \\
\text { Placebo arm (n): } 28 \\
\text { Average age of vitamin D and calcium arm: } 28.7 \pm 6.0 \\
\text { years } \\
\text { Average age of placebo arm: } 30.8 \pm 6.6 \text { years } \\
\text { Missing outcome data: } 5 \\
\text { Baseline mean (SD) GW: Vitamin D and calcium arm: } \\
73.6 \pm 13.0 \mathrm{~kg} ; \text { placebo arm: } 78.2 \pm 13.6 \mathrm{~kg} \\
\text { Baseline mean (SD) BMI: Vitamin D and calcium arm: } \\
29.4 \pm 4.6 \mathrm{~kg} / \mathrm{m}^{2} ; \text { placebo arm: } 30.5 \pm 4.6 \mathrm{~kg} / \mathrm{m}^{2} \\
\text { Baseline mean (SD) } 25(\mathrm{OH}) \mathrm{D}: \text { Vitamin D and calcium } \\
\text { arm: } 43.11 \pm 28.17 \mathrm{nmol} / \mathrm{L} ; \text { placebo arm: } 49.05 \pm 34.30 \\
\mathrm{nmol} / \mathrm{L}\end{array}$ & $\begin{array}{l}\text { Vitamin D and calcium } \\
\text { arm: } 1,000 \text { mg calcium } \\
\text { carbonate (daily) and } \\
50,000 \mathrm{U} \text { D3 (at trial } \\
\text { initiation and on } 21^{\mathrm{st}} \\
\text { day) } \\
\text { Placebo arm. } \\
\text { Total vitamin D received } \\
\text { in six weeks: } 100,000 \mathrm{IU}\end{array}$ & $\begin{array}{l}\text { 1. GW } \\
\text { 2. BMI } \\
\text { 3. } 25(\mathrm{OH}) \mathrm{D}\end{array}$ \\
\hline
\end{tabular}


Table 1. Continued

\begin{tabular}{|c|c|c|c|c|}
\hline Trial & Design & Population & Intervention arms & $\begin{array}{l}\text { Outcomes } \\
\text { reported }\end{array}$ \\
\hline $\begin{array}{l}\text { Jamilian } \\
\text { et al. (15) }\end{array}$ & $\begin{array}{l}\text { Randomized placebo-controlled } \\
\text { clinical trial } \\
\text { Blinding: double blinded } \\
\text { No of treatment arms: two } \\
\text { Single centered trial } \\
\text { Study duration: six weeks. } \\
\text { Country where trial was } \\
\text { conducted: Iran } \\
\text { Ethical permission: obtained } \\
\text { Consent from participants: } \\
\text { obtained } \\
\text { Information regarding funding: } \\
\text { provided } \\
\text { Clinical trial registration number: } \\
\text { IRCT201509115623N52 }\end{array}$ & $\begin{array}{l}\text { Diagnosis: GDM (using ADA criteria) } \\
\text { Number of participants randomized: } 60 \\
\text { Vitamin D3 and EPO arm (n): } 30 \\
\text { Placebo arm (n): } 30 \\
\text { Average age of vitamin D3 and EPO arm: } 28.4 \pm 6.2 \\
\text { years } \\
\text { Average age of placebo arm: } 29.6 \pm 4.3 \text { years } \\
\text { Missing outcome data: } 6 \text { (all withdrawn from the } \\
\text { trial due to personal reasons) } \\
\text { Baseline mean (SD) GW: Vitamin D3 and EPO arm: } \\
71.5 \pm 10.8 \mathrm{~kg} ; \text { placebo arm: } 72.3 \pm 8.5 \mathrm{~kg} \\
\text { Baseline mean (SD) BMI: Vitamin D3 and EPO arm: } \\
27.0 \pm 4.2 \mathrm{~kg} / \mathrm{m}^{2} ; \text { placebo arm: } 27.6 \pm 3.5 \mathrm{~kg} / \mathrm{m}^{2} \\
\text { Baseline mean (SD) } 25(\mathrm{OH}) \mathrm{D}: \text { Vitamin D3 and EPO } \\
\text { arm: } 14.0 \pm 10.1 \mathrm{ng} / \mathrm{mL} \text {; placebo arm: } 11.4 \pm 4.3 \mathrm{ng} / \\
\mathrm{mL}\end{array}$ & $\begin{array}{l}\text { Vitamin D3 and EPO } \\
\text { arm: 1,000 IU of vitamin } \\
\text { D and 1,000 mg of EPO: } \\
\text { daily (60) } \\
\text { Placebo arm. } \\
\text { Total vitamin D received } \\
\text { in six weeks: } 42,000 \mathrm{IU} .\end{array}$ & $\begin{array}{l}\text { 1. GW } \\
\text { 2. BMI } \\
\text { 3. } 25(\mathrm{OH}) \mathrm{D}\end{array}$ \\
\hline $\begin{array}{l}\text { Jamilian } \\
\text { et al. (35) }\end{array}$ & $\begin{array}{l}\text { Randomized, placebo-controlled } \\
\text { clinical trial } \\
\text { Blinding: double blinded } \\
\text { No. of treatment arms: four } \\
\text { Single centered trial } \\
\text { Study duration: six weeks. } \\
\text { Country where trial was } \\
\text { conducted: Iran } \\
\text { Ethical permission: obtained } \\
\text { Consent from participants: } \\
\text { obtained } \\
\text { Information regarding funding: } \\
\text { provided } \\
\text { Clinical trial registration number: } \\
\text { IRCT201605135623N78 }\end{array}$ & $\begin{array}{l}\text { Diagnosis: GDM (using ADA criteria) } \\
\text { Number of participants randomized: } 140 \\
\text { Vitamin D and omega-3 fatty acid arm (n): } 35 \\
\text { Vitamin D arm (n): } 35 \\
\text { Omeag-3 fatty acid arm (n): } 35 \\
\text { Placebo arm (n): } 35 \\
\text { Average age of vitamin D and omega-3 fatty acid } \\
\text { arm: } 31.2 \pm 4.3 \text { years } \\
\text { Average age of vitamin D arm: } 31.5 \pm 7.0 \text { years } \\
\text { Average age of omega-3 arm: } 30.7 \pm 3.5 \text { years } \\
\text { Average age of placebo arm: } 30.7 \pm 4.1 \text { years } \\
\text { Missing outcome data: } 6 \text { (all withdrawn from the } \\
\text { trial due to personal reasons) } \\
\text { Baseline mean (SD) GW: } \\
\text { Vitamin D and omega- } 3 \mathrm{fatty} \text { acid arm: } 77.3 \pm 9.9 \mathrm{~kg} \\
\text { Vitamin D arm: } 78.4 \pm 15.2 \mathrm{~kg} \\
\text { Omeag-3 fatty acid arm: } 75.0 \pm 5.8 \mathrm{~kg} \\
\text { Placebo arm: } 75.9 \pm 7.1 \mathrm{~kg} \\
\text { Baseline mean (SD) BMI: Vitamin D and omega-3 } \\
\text { fatty acid arm: } 29.7 \pm 3.9 \mathrm{~kg} / \mathrm{m}^{2} \\
\text { Vitamin D arm: } 29.7 \pm 5.1 \mathrm{~kg} / \mathrm{m}^{2} \\
\text { Omeag-3 fatty acid arm: } 28.8 \pm 2.4 \mathrm{~kg} / \mathrm{m}^{2} \\
\text { Placebo arm: } 29.2 \pm 3.4 \mathrm{~kg} / \mathrm{m}^{2} \\
\text { Baseline mean (SD) } 25(\mathrm{OH}) \mathrm{D}: \mathrm{Vitamin} \mathrm{D} \text { and } \\
\text { omega-3 fatty acid arm: } 15.5 \pm 3.1 \mathrm{ng} / \mathrm{mL} ; \text { vitamin } \\
\text { D arm: } 15.2 \pm 3.8 \mathrm{ng} / \mathrm{mL} ; \mathrm{Omeag}-3 \mathrm{fatty} \text { acid } \mathrm{arm}: \\
16.9 \pm 3.5 \mathrm{ng} / \mathrm{mL} ; \mathrm{placebo} \text { arm: } 16.6 \pm 2.6 \mathrm{ng} / \mathrm{mL}\end{array}$ & $\begin{array}{l}\text { Vitamin D and omega-3 } \\
\text { fatty acid arm: } 50,000 \\
\text { IU of vitamin D (two } \\
\text { weekly) and } 1000 \mathrm{mg} \\
\text { omega-3 fatty acid } \\
\text { (twice daily) } \\
\text { Vitamin D arm: } 50000 \mathrm{IU} \\
\text { vitamin D (two weekly) } \\
\text { Omega-3 fatty acid arm: } \\
1000 \text { mg omega-3 fatty } \\
\text { acids } \\
\text { Placebo arm. } \\
\text { Total vitamin D received } \\
\text { in six weeks: } 150,000 \mathrm{IU}\end{array}$ & $\begin{array}{l}\text { 1. GW } \\
\text { 2. BMI } \\
\text { 3. } 25(\mathrm{OH}) \mathrm{D}\end{array}$ \\
\hline
\end{tabular}

ADA: American Diabetes Association (52,53); EPO: evening primrose oil, GDM: Gestational diabetes mellitus, BMI: Body mass index, SD: Standard deviation, GW: Gestation weight, 25(OH)D: 25-hydroxyvitamin D 


\section{Implications and strengths}

The chief inference of this paper is that it informs about the rigor of the current evidence of the maternal benefits of prenatal vitamin D supplementation in GDM patients. From the perspective of maternal health, this study may help health authorities to determine if large scale supplementation for all GDM pregnancies will be an appropriate public health initiative or not, given the current evidence. Moreover, as the reviewed trials were primarily Iran-based, this paper might encourage

Table 2. Risk of bias assessment

\begin{tabular}{|c|c|c|c|c|c|c|c|}
\hline Trial $^{\#}$ & $\begin{array}{l}\text { Selection } \\
\text { bias } \\
\text { (Random } \\
\text { sequence } \\
\text { generation) }\end{array}$ & $\begin{array}{l}\text { Selection bias } \\
\text { (Allocation } \\
\text { concealment) }\end{array}$ & $\begin{array}{l}\text { Performance } \\
\text { bias } \\
\text { Outcome: } \\
\text { BMI, GW, and } \\
\text { 25(OH)2D }\end{array}$ & $\begin{array}{l}\text { Detection } \\
\text { bias } \\
\text { Outcome: } \\
\text { BMI, GW, and } \\
\text { 25(OH)2D }\end{array}$ & $\begin{array}{l}\text { Attrition } \\
\text { bias }\end{array}$ & $\begin{array}{l}\text { Reporting } \\
\text { bias }\end{array}$ & $\begin{array}{l}\text { Other } \\
\text { bias }\end{array}$ \\
\hline \multirow[b]{2}{*}{ Karamali et al. (16) } & Low & Unclear & Unclear & Low & Low & Low & Low \\
\hline & \multicolumn{7}{|c|}{$\begin{array}{l}\text { Authors' comment: Allocation concealment: it's not clear if the midwife who measured did the random allocation } \\
\text { of participant (in an unblind manner) was related the study personnel or the outcome assessor; Performance } \\
\text { bias: Participants were blinded by making the placebos identical to the supplements. However, it's not clear if } \\
\text { study personnel were adequately blinded or not. }\end{array}$} \\
\hline \multirow[b]{2}{*}{ Karamali et al.(17) } & Low & Unclear & Unclear & Low & Low & Low & Low \\
\hline & \multicolumn{7}{|c|}{$\begin{array}{l}\text { Authors' comment: Allocation concealment: Precise mechanism not clear; Performance bias: Participants were } \\
\text { blinded by making the placebos identical to the supplements. However, it's not clear if study personnel were } \\
\text { adequately blinded or not. }\end{array}$} \\
\hline \multirow[b]{2}{*}{ Jamilian et al. (50) } & Low & Unclear & Unclear & Low & Low & Low & Low \\
\hline & \multicolumn{7}{|c|}{$\begin{array}{l}\text { Authors' comment: Allocation concealment: Precise mechanism not clear; Performance bias: Participants were } \\
\text { blinded by making the placebos identical to the supplements. However, it's not clear if study personnel were } \\
\text { adequately blinded or not. }\end{array}$} \\
\hline \multirow[b]{2}{*}{ Jamilian et al. (18) } & Low & Low & Unclear & Low & Low & Low & Low \\
\hline & \multicolumn{7}{|c|}{$\begin{array}{l}\text { Authors' comment: Performance bias: Participants were blinded by making the placebos identical to the } \\
\text { supplements. However, it's not clear if study personnel were adequately blinded or not. }\end{array}$} \\
\hline \multirow[b]{2}{*}{ Li and Xing (37) } & Low & Unclear & Unclear & Low & Low & Low & Low \\
\hline & \multicolumn{7}{|c|}{$\begin{array}{l}\text { Authors' comment: Participants were blinded by using coded labels on the interventions. However, it remains } \\
\text { unclear if study personnel were adequately blinded or not. }\end{array}$} \\
\hline Yazdchi et al. (33) & \multicolumn{7}{|c|}{$\begin{array}{l}\text { Authors' comment: Allocation concealment: Precise mechanism not clear; Performance bias: Participants were } \\
\text { blinded by making the placebos identical to the supplements. However, it's not clear if study personnel were } \\
\text { adequately blinded or not. }\end{array}$} \\
\hline Asemi et al. (34) & Low & Low & Low & Low & Low & Low & Low \\
\hline \multirow[b]{2}{*}{ Jamilian et al. (15) } & Low & Unclear & Unclear & Low & Low & Low & Low \\
\hline & \multicolumn{7}{|c|}{$\begin{array}{l}\text { Authors' comment: It remains unclear if the midwife responsible for random sequence generation and its } \\
\text { allocation concealment was also the study personnel or anyway could have broken the blinding of the study } \\
\text { personnel. }\end{array}$} \\
\hline \multirow[b]{2}{*}{ Jamilian et al. (35) } & Low & Unclear & Unclear & Unclear & Low & Low & Low \\
\hline & \multicolumn{7}{|c|}{$\begin{array}{l}\text { Authors' comment: The precise mechanism used to keep the allocation sequence of the computer-generated } \\
\text { random numbers concealed from the participants was not clear. It's not clear how were study personnel } \\
\text { and participants blinded in this trial as we couldn't find a clear mention about it. It also remains unclear if the } \\
\text { nutritionist and the midwife measuring weight and height of participants were part of the intervention providing } \\
\text { team or anyway their blinding might have been broken about the interventions received by the participants. }\end{array}$} \\
\hline
\end{tabular}




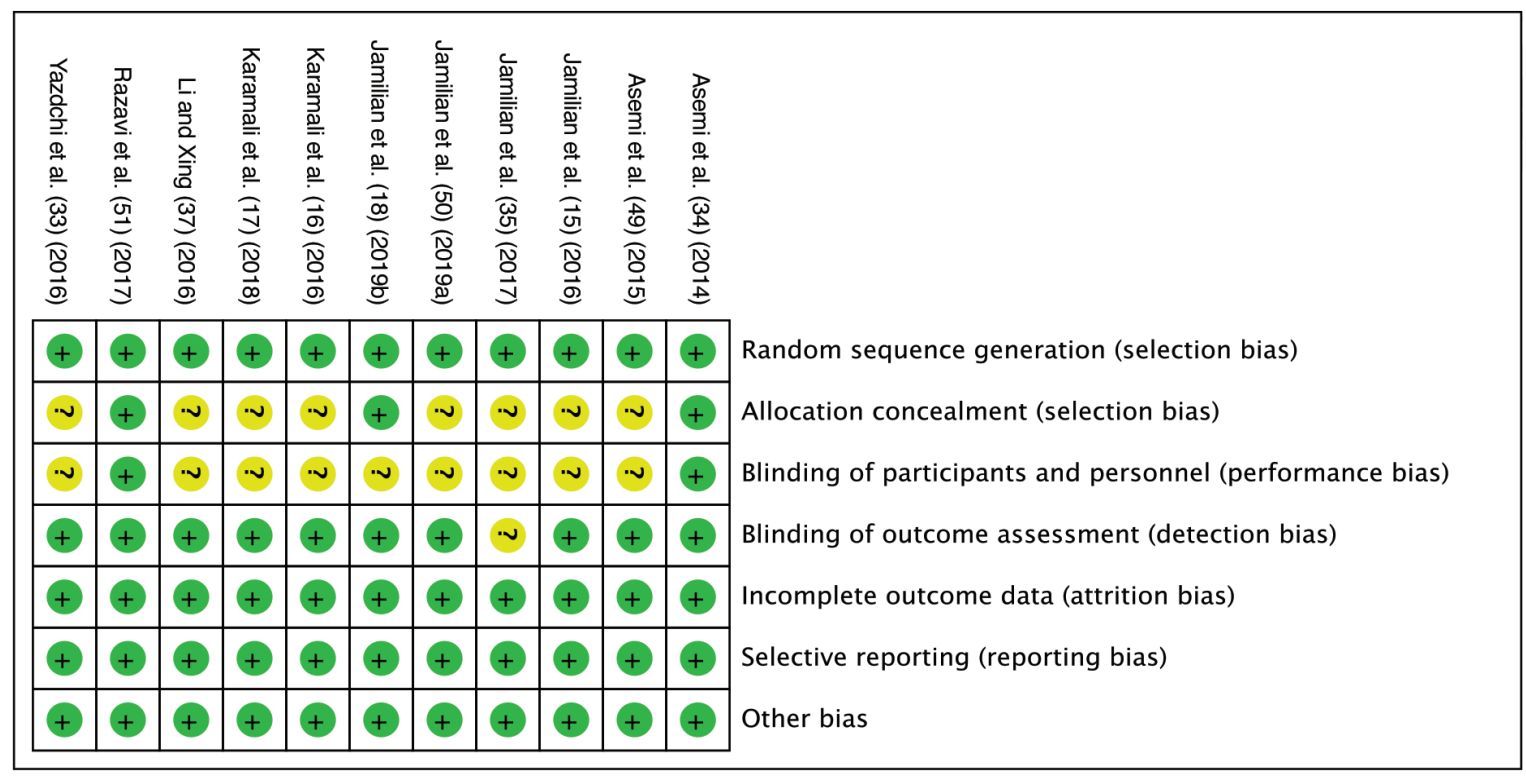

$2 a$.

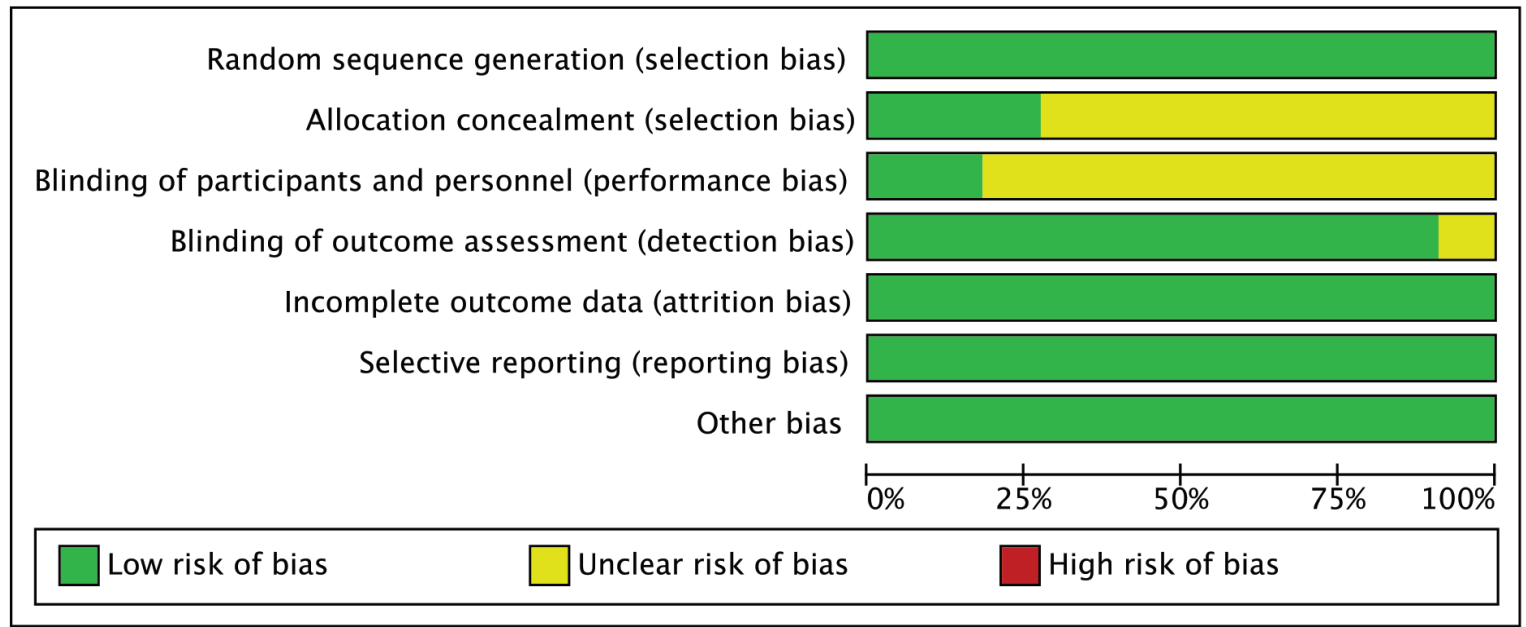

$2 b$.

Figure 2. a) Risk of bias graph: review authors' evaluation of respective risk of bias items presented across all studies included in the review. b) Risk of bias summary: review authors' evaluation of respective risk of bias item for each included study

future trialists to conduct identical trials globally to generate generalizable evidence. Concerning the strength, this systematic review is one of the preliminary efforts to investigate the maternal effects of antenatal vitamin D supplementation in GDM patients. A further strength was the unbound nature of our electronic database searche to include any date, language, or geographical boundary, thus adding comprehensiveness to our review. Finally, evidence generated from this systematic review and metaanalysis is likely to be rigorous, as it's grounded on the highest level of epidemiological evidence, randomized controlled trials. Despite these strengths, this paper has a few weaknesses. As most trials were conducted in Iran, the external validity of this review is likely to be compromised. The heterogeneity observed for the $25(\mathrm{OH}) \mathrm{D}$ levels might have increased the risk of bias in our estimates, and this can be because one of the studies was not from Iran. Besides, the maternal health effects of vitamin D supplementation remains inseparable from other supplements that were simultaneously given to the participants in most trials.

\section{Conclusion}

Using vitamin $\mathrm{D}$ as the chief ingredient of antenatal supplements favors in blood 25(OH)D level rise in GDM patients. However, the effect of these supplements on GW and BMI was not distinguishable from those subjects who did not receive supplementation. 


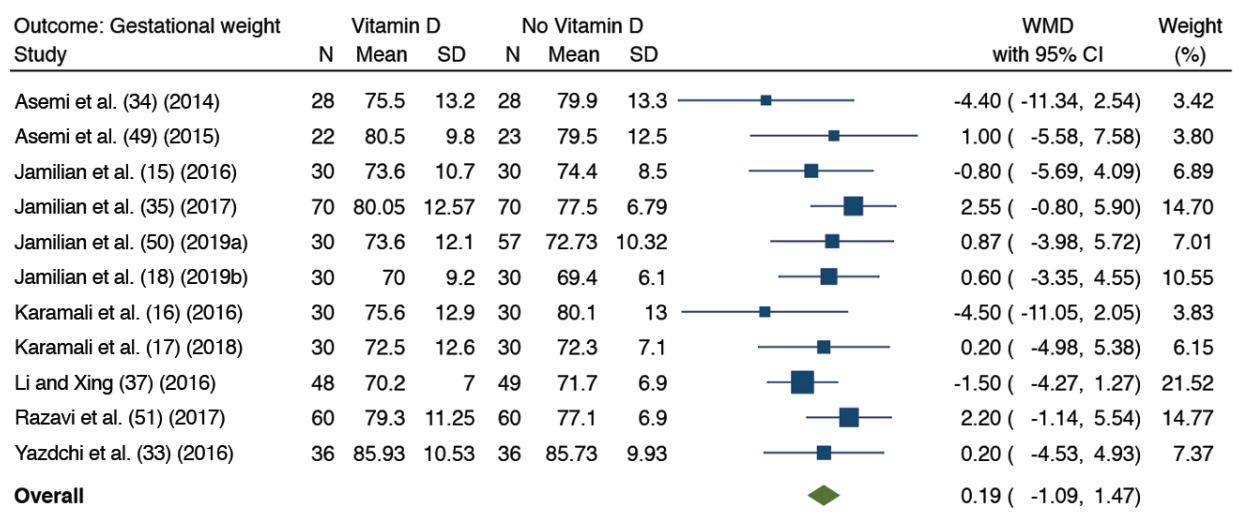

Heterogeneity: $\mathrm{T}^{2}=0.00, \mathrm{I}^{2}=0.00 \%, \mathrm{H}^{2}=1.00$

Test of $\theta_{\mathrm{i}}=\theta_{\mathrm{i}}: \mathrm{Q}(10)=8.72, \mathrm{p}=0.56$

$3 a$

Test of $\theta=0: z=0.29, p=0.77$

Random-effects DerSimonian-Laird model

\begin{tabular}{|c|c|c|c|c|c|c|c|c|}
\hline \multirow{2}{*}{$\begin{array}{l}\text { Outcome: Body mass index } \\
\text { Study }\end{array}$} & \multicolumn{3}{|c|}{ Vitamin D } & \multicolumn{3}{|c|}{ No Vitamin D } & \multirow{2}{*}{$\begin{array}{c}\text { WMD } \\
\text { with } 95 \% \mathrm{Cl} \\
\end{array}$} & \multirow{2}{*}{$\begin{array}{l}\text { Weight } \\
(\%)\end{array}$} \\
\hline & $\mathrm{N}$ & Mean & SD & $\mathrm{N}$ & Mean & SD & & \\
\hline Asemi et al. (34) (2014) & 28 & 30.2 & 4.7 & 28 & 31.2 & 4.5 & $-1.00(-3.41,1.41)$ & 5.17 \\
\hline Asemi et al. (49) (2015) & 22 & 31.2 & 4 & 23 & 31.1 & 4.3 & $0.10(-2.33,2.53)$ & 5.09 \\
\hline Jamilian et al. (15) (2016) & 30 & 27.7 & 4.2 & 30 & 28.4 & 3.3 & $-0.70(-2.61,1.21)$ & 8.23 \\
\hline Jamilian et al. (35) (2017) & 70 & 30.5 & 4.45 & 70 & 29.75 & 2.93 & $0.75(-0.50,2.00)$ & 19.29 \\
\hline Jamilian et al. (50) (2019a) & 30 & 28.5 & 4.9 & 57 & 27.59 & 3.74 & $0.91(-0.93,2.75)$ & 8.83 \\
\hline Jamilian et al. (18) (2019b) & 30 & 26.4 & 3.6 & 30 & 26 & 2.6 & $0.40(-1.19,1.99)$ & 11.90 \\
\hline Karamali et al. (16) (2016) & 30 & 30.2 & 4.7 & 30 & 31.2 & 4.4 & $-1.00(-3.30,1.30)$ & 5.66 \\
\hline Karamali et al. (17) (2018) & 30 & 28 & 4.7 & 30 & 27.6 & 2.6 & $0.40(-1.52,2.32)$ & 8.13 \\
\hline Razavi et al. (51) (2017) & 60 & 30.3 & 4.25 & 60 & 29.55 & 2.96 & $0.75(-0.56,2.06)$ & 17.50 \\
\hline Yazdchi et al. (33) (2016) & 36 & 33.24 & 3.63 & 36 & 33.28 & 3.8 & $-0.04(-1.76,1.68)$ & 10.20 \\
\hline Overall & & & & & & & $0.27(-0.28,0.82)$ & \\
\hline
\end{tabular}

Heterogeneity: $\mathrm{T}^{2}=0.00, \mathrm{I}^{2}=0.00 \%, \mathrm{H}^{2}=1.00$

Test of $\theta_{i}=\theta_{i}: Q(9)=4.96, p=0.84$

Test of $\theta=0: z=0.97, p=0.33$

Random-effects DerSimonian-Laird model

\begin{tabular}{|c|c|c|c|c|c|c|c|c|}
\hline \multirow{2}{*}{$\begin{array}{l}\text { Outcome: Blood 25(OH)D level } \\
\text { Study }\end{array}$} & \multicolumn{3}{|c|}{ Vitamin D } & \multicolumn{3}{|c|}{ No Vitamin D } & \multirow{2}{*}{$\begin{array}{c}\text { SMD } \\
\text { with } 95 \% \mathrm{Cl}\end{array}$} & \multirow{2}{*}{$\begin{array}{l}\text { Weight } \\
(\%)\end{array}$} \\
\hline & $\mathrm{N}$ & Mean & SD & $\mathrm{N}$ & Mean & SD & & \\
\hline Asemi et al. (34) (2014) & 28 & 91.3 & 54.6 & 28 & 50.8 & 35.48 & $0.87(0.33,1.41)$ & 10.05 \\
\hline Asemi et al. (49) (2015) & 22 & 40.4 & 27 & 23 & 21.5 & 14.8 & $0.86(0.26,1.46)$ & 9.97 \\
\hline Jamilian et al. (15) (2016) & 30 & 20.9 & 10.3 & 30 & 11.3 & 4.7 & $1.18(0.64,1.73)$ & 10.05 \\
\hline Jamilian et al. (35) (2017) & 70 & 35.75 & 5.3 & 70 & 17.15 & 3.24 & $4.21(3.62,4.80)$ & 9.98 \\
\hline Jamilian et al. (50) (2019a) & 30 & 35.1 & 3.9 & 57 & 18.11 & 3.4 & $4.71(3.88,5.53)$ & 9.60 \\
\hline Jamilian et al. (18) (2019b) & 30 & 18.7 & 4.7 & 30 & 17.3 & 3.1 & $0.35(-0.16,0.85)$ & 10.10 \\
\hline Karamali et al. (16) (2016) & 30 & 36.3 & 21.3 & 30 & 21.3 & 14.4 & $0.81(0.29,1.33)$ & 10.08 \\
\hline Karamali et al. (17) (2018) & 30 & 32.44 & 16.72 & 30 & 20.71 & 11.23 & $0.81(0.29,1.33)$ & 10.08 \\
\hline Li and Xing (37) (2016) & 48 & 29.5 & 5.7 & 49 & 15.9 & 4.5 & $2.63(2.09,3.17)$ & 10.05 \\
\hline \multirow[t]{2}{*}{ Razavi et al. (51) (2017) } & 60 & 32.75 & 5.85 & 60 & 15.5 & 4.01 & $3.42(2.86,3.98)$ & 10.03 \\
\hline & & & & & & & $1.97(1.06,2.88)$ & \\
\hline
\end{tabular}

Heterogeneity: $\mathrm{t}^{2}=2.08, \mathrm{I}^{2}=96.21 \%, \mathrm{H}^{2}=26.37$

Test of $\theta_{\mathrm{i}}=\theta_{\mathrm{j}}: \mathrm{Q}(9)=237.29, \mathrm{p}=0.00$

Test of $\theta=0: z=4.23, p=0.00$

Random-effects DerSimonian-Laird model

Figure 3. Forest plots depicting meta-analysis findings (random-effect model). Outcome: gestational weight a), body mass index b), and 25(OH)D level in blood c). A comparison between antenatal vitamin D supplementation (as the only or cosupplement with other supplements) and non-vitamin D based supplementation; Two trials had with identical trial author name and year have been suffixed with alphabet " $a$ " $(50)$ and " $b$ " (18) after the study name and year 


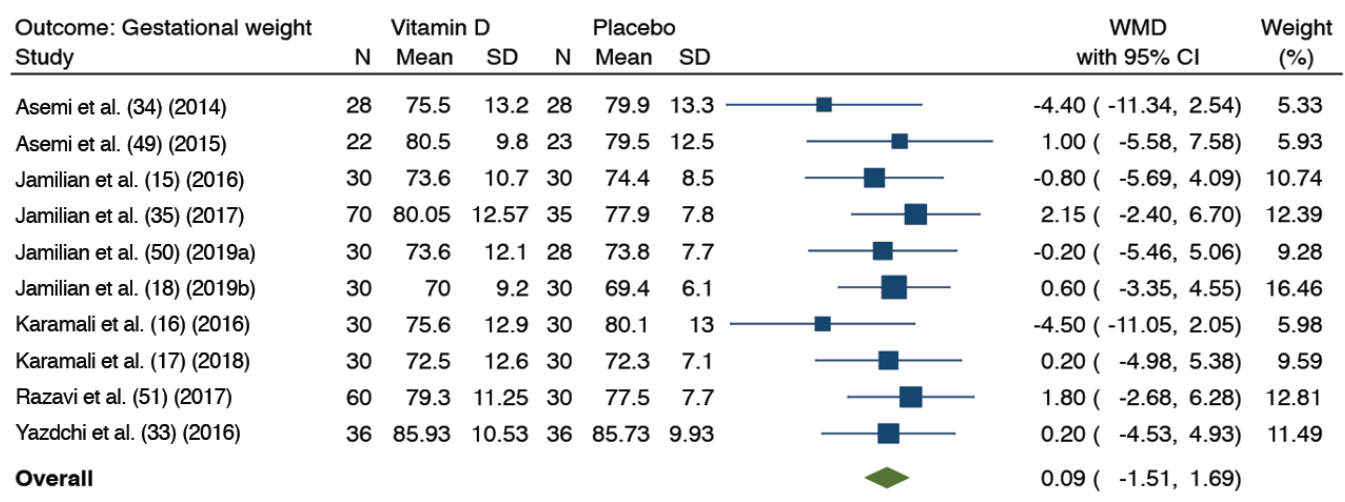

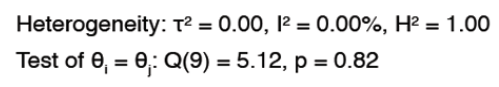

Random-effects DerSimonian-Laird model

\begin{tabular}{|c|c|c|c|c|c|c|c|c|}
\hline \multirow{2}{*}{$\begin{array}{l}\text { Outcome: Body mass index } \\
\text { Study }\end{array}$} & \multicolumn{3}{|c|}{ Vitamin D } & \multicolumn{3}{|c|}{ Placebo } & \multirow{2}{*}{$\begin{array}{c}\text { WMD } \\
\text { with } 95 \% \mathrm{Cl}\end{array}$} & \multirow{2}{*}{$\begin{array}{l}\text { Weight } \\
(\%)\end{array}$} \\
\hline & $\mathrm{N}$ & Mean & SD & $\mathrm{N}$ & Mean & SD & & \\
\hline Asemi et al. (34) (2014) & 28 & 30.2 & 4.7 & 28 & 31.2 & 4.5 & $-1.00(-3.41,1.41)$ & 6.36 \\
\hline Asemi et al. (49) (2015) & 22 & 31.2 & 4 & 23 & 31.1 & 4.3 & $0.10(-2.33,2.53)$ & 6.26 \\
\hline Jamilian et al. (15) (2016) & 30 & 27.7 & 4.2 & 30 & 28.4 & 3.3 & $-0.70(-2.61,1.21)$ & 10.12 \\
\hline Jamilian et al. (35) (2017) & 70 & 30.5 & 4.45 & 35 & 29.9 & 3.4 & $0.60(-1.08,2.28)$ & 13.14 \\
\hline Jamilian et al. (50) (2019a) & 30 & 28.5 & 4.9 & 28 & 28.2 & 3.3 & $0.30(-1.87,2.47)$ & 7.88 \\
\hline Jamilian et al. (18) (2019b) & 30 & 26.4 & 3.6 & 30 & 26 & 2.6 & $0.40(-1.19,1.99)$ & 14.64 \\
\hline Karamali et al. (16) (2016) & 30 & 30.2 & 4.7 & 30 & 31.2 & 4.4 & $-1.00(-3.30,1.30)$ & 6.96 \\
\hline Karamali et al. (17) (2018) & 30 & 28 & 4.7 & 30 & 27.6 & 2.6 & $0.40(-1.52,2.32)$ & 10.00 \\
\hline Razavi et al. (51) (2017) & 60 & 30.3 & 4.25 & 30 & 29.7 & 3.4 & $0.60(-1.15,2.35)$ & 12.09 \\
\hline Yazdchi et al. (33) (2016) & 36 & 33.24 & 3.63 & 36 & 33.28 & 3.8 & $-0.04(-1.76,1.68)$ & 12.54 \\
\hline Overall & & & & & & & $0.07(-0.54,0.68)$ & \\
\hline
\end{tabular}

Heterogeneity: $\mathrm{t}^{2}=0.00, \mathrm{I}^{2}=0.00 \%, \mathrm{H}^{2}=1.00$

Test of $\theta_{i}=\theta_{j}: Q(9)=3.28, p=0.95$

Test of $\theta=0: z=0.23, p=0.82$

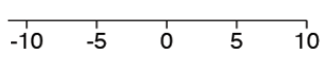

a

Random-effects DerSimonian-Laird model

\begin{tabular}{|c|c|c|c|c|c|c|c|c|}
\hline \multirow{2}{*}{$\begin{array}{l}\text { Outcome: Blood 25(OH)D level } \\
\text { Study }\end{array}$} & \multicolumn{3}{|c|}{ Vitamin D } & \multicolumn{3}{|c|}{ Placebo } & \multirow{2}{*}{$\begin{array}{c}\text { SMD } \\
\text { with } 95 \% \mathrm{Cl}\end{array}$} & \multirow{2}{*}{$\begin{array}{l}\text { Weight } \\
(\%)\end{array}$} \\
\hline & $\mathrm{N}$ & Mean & SD & $\mathrm{N}$ & Mean & SD & & \\
\hline Asemi et al. (34) (2014) & 28 & 91.3 & 54.6 & 28 & 50.8 & 35.48 & $0.87(0.33,1.41)$ & 11.26 \\
\hline Asemi et al. (49) (2015) & 22 & 40.4 & 27 & 23 & 21.5 & 14.8 & $0.86(0.26,1.46)$ & 11.16 \\
\hline Jamilian et al. (15) (2016) & 30 & 20.9 & 10.3 & 30 & 11.3 & 4.7 & $1.18(0.64,1.73)$ & 11.26 \\
\hline Jamilian et al. (35) (2017) & 70 & 35.75 & 5.3 & 35 & 16.5 & 2.6 & $4.17(3.47,4.86)$ & 10.99 \\
\hline Jamilian et al. (50) (2019a) & 30 & 35.1 & 3.9 & 28 & 17.7 & 3.7 & $4.51(3.55,5.48)$ & 10.37 \\
\hline Jamilian et al. (18) (2019b) & 30 & 18.7 & 4.7 & 30 & 17.3 & 3.1 & $0.35(-0.16,0.85)$ & 11.33 \\
\hline Karamali et al. (16) (2016) & 30 & 36.3 & 21.3 & 30 & 21.3 & 14.4 & $0.81(0.29,1.33)$ & 11.30 \\
\hline Karamali et al. (17) (2018) & 30 & 32.44 & 16.72 & 30 & 20.71 & 11.23 & $0.81(0.29,1.33)$ & 11.30 \\
\hline Razavi et al. (51) (2017) & 60 & 32.75 & 5.85 & 30 & 14.7 & 3.2 & $3.49(2.82,4.16)$ & 11.03 \\
\hline Overall & & & & & & & $1.86(0.95,2.77)$ & \\
\hline
\end{tabular}

Heterogeneity: $\mathrm{\tau}^{2}=1.85, \mathrm{I}^{2}=95.36 \%, \mathrm{H}^{2}=21.54$

Test of $\theta_{i}=\theta_{j}: Q(8)=172.31, p=0.00$

Test of $\theta=0: z=3.99, p=0.00$

Random-effects DerSimonian-Laird model

Figure 4. Forest plots depicting meta-analysis findings (random-effect model). Outcome: gestational weight a), body mass index b), and 25(OH)D level in blood c). A comparison between antenatal vitamin D supplementation (as a sole or cosupplement with other supplements) and placebo; Two trials had with identical trial author name and year have been suffixed with alphabet "a" (50) and "b" (18) after the study name and year 


\section{Peer-review: Externally peer-reviewed.}

\section{Conflict of Interest: No conflict of interest is declared by the} authors.

\section{Financial Disclosure: The authors declared that this study} received no financial support.

\section{References}

1. Quintanilla Rodriguez BS, Mahdy H. Gestational Diabetes. [Updated: 2019 Dec 23]. In: StatPearls [Internet] Treasure Island (FL): StatPearls Publishing; 2020. Available from: https://www.ncbi. nlm.nih.gov/books/NBK545196/

2. Xiong X, Saunders LD, Wang FL, Demianczuk NN. Gestational diabetes mellitus: prevalence, risk factors, maternal and infant outcomes. Int J Gynaecol Obstet 2001; 75: 221-8.

3. Nguyen CL, Pham NM, Binns CW, Duong D Van, Lee AH. Prevalence of Gestational Diabetes Mellitus in Eastern and Southeastern Asia: A Systematic Review and Meta-Analysis. J Diabetes Res 2018; 2018 : 6536974.

4. Ferrara A. Increasing prevalence of gestational diabetes mellitus: a public health perspective. Diabetes Care 2007; 30(Suppl 2): S141-6.

5. Adam S, Rheeder P. Screening for gestational diabetes mellitus in a South African population: Prevalence, comparison of diagnostic criteria and the role of risk factors. S Afr Med J 2017; 107: 523-7.

6. Gou BH, Guan HM, Bi YX, Ding BJ. Gestational diabetes: weight gain during pregnancy and its relationship to pregnancy outcomes. Chin Med J 2019; 132: 154-60.

7. Macrì F, Pitocco D, di Pasquo E, Salvi S, Rizzi A, Di Leo M, et al. Gestational weight gain as an independent risk factor for adverse pregnancy outcomes in women with gestational diabetes. Eur Rev Med Pharmacol Sci 2018; 22: 4403-10.

8. Gante I, Amaral N, Dores J, Almeida MC. Impact of gestational weight gain on obstetric and neonatal outcomes in obese diabetic women. BMC Pregnancy Childbirth 2015; 15: 249.

9. Liu L, Hong Z, Zhang L. Associations of prepregnancy body mass index and gestational weight gain with pregnancy outcomes in nulliparous women delivering single live babies. Sci Rep 2015; 5 12863.

10. Egan AM, Dennedy MC, Al-Ramli W, Heerey A, Avalos G, Dunne F. ATLANTIC-DIP: excessive gestational weight gain and pregnancy outcomes in women with gestational or pregestational diabetes mellitus. J Clin Endocrinol Metab 2014; 99: 212-9.

11. Kim SY, Sharma AJ, Sappenfield W, Wilson HG, Salihu HM. Association of maternal body mass index, excessive weight gain, and gestational diabetes mellitus with large-for-gestational-age births. Obstet Gynecol 2014; 123: 737-44.

12. IOM (Institute of Medicine) and NRC (National Research Council). Weight Gain During Pregnancy. In: Rasmussen KM, Yaktine AL editors. Washington DC, Washington (DC): National Academies Press; 2009.

13. Horosz E, Bomba-Opon DA, Szymanska M, Wielgos M. Maternal weight gain in women with gestational diabetes mellitus. J Perinat Med 2013; 41: 523-8.

14. Park JE, Park S, Daily JW, Kim SH. Low gestational weight gain improves infant and maternal pregnancy outcomes in overweight and obese Korean women with gestational diabetes mellitus. Gynecol Endocrinol 2011; 27: 775-81.

15. Jamilian M, Karamali M, Taghizadeh M, Sharifi N, Jafari Z, Memarzadeh MR, et al. Vitamin D and Evening Primrose Oil
Administration Improve Glycemia and Lipid Profiles in Women with Gestational Diabetes. Lipids 2016; 51: 349-56.

16. Karamali M, Asemi Z, Ahmadi-Dastjerdi M, Esmaillzadeh A. Calcium plus vitamin D supplementation affects pregnancy outcomes in gestational diabetes: randomized, double-blind, placebo-controlled trial. Public Health Nutr 2016; 19: 156-63.

17. Karamali M, Bahramimoghadam S, Sharifzadeh F, Asemi Z. Magnesium-zinc-calcium-vitamin D co-supplementation improves glycemic control and markers of cardiometabolic risk in gestational diabetes: a randomized, double-blind, placebo-controlled trial. Appl Physiol Nutr Metab 2018; 43: 565-70.

18. Jamilian M, Mirhosseini N, Eslahi M, Bahmani F, Shokrpour M, Chamani M, et al. The effects of magnesium-zinc-calcium-vitamin D co-supplementation on biomarkers of inflammation, oxidative stress and pregnancy outcomes in gestational diabetes. BMC Pregnancy Childbirth 2019; 19: 107.

19. Aghajafari F, Nagulesapillai T, Ronksley PE, Tough SC, O'Beirne M, Rabi DM. Association between maternal serum 25-hydroxyvitamin D level and pregnancy and neonatal outcomes: systematic review and meta-analysis of observational studies. BMJ 2013; 346: f1169.

20. Lu M, Xu Y, Lv L, Zhang M. Association between vitamin D status and the risk of gestational diabetes mellitus: a meta-analysis. Arch Gynecol Obstet 2016; 293: 959-66.

21. Poel YH, Hummel P, Lips P, Stam F, van der Ploeg T, Simsek S. Vitamin $\mathrm{D}$ and gestational diabetes: a systematic review and metaanalysis. Eur J Intern Med 2012; 23: 465-9.

22. Wei SQ, Qi HP, Luo ZC, Fraser WD. Maternal vitamin D status and adverse pregnancy outcomes: a systematic review and metaanalysis. J Matern Fetal Neonatal Med 2013; 26: 889-99.

23. Zhang MX, Pan GT, Guo JF, Li BY, Qin LQ, Zhang ZL. Vitamin D Deficiency Increases the Risk of Gestational Diabetes Mellitus: A Meta-Analysis of Observational Studies. Nutrients 2015; 07: 8366-75.

24. Spaight C, Gross J, Horsch A, Puder JJ. Gestational Diabetes Mellitus. Endocr Dev 2016; 31: 163-78.

25. Sablok A, Batra A, Thariani K, Batra A, Bharti R, Aggarwal AR, et al. Supplementation of vitamin D in pregnancy and its correlation with feto-maternal outcome. Clin Endocrinol (Oxf) 2015; 83: 536-41.

26. Rostami M, Tehrani FR, Simbar M, Bidhendi Yarandi R, Minooee S, Hollis BW, et al. Effectiveness of Prenatal Vitamin D Deficiency Screening and Treatment Program: A Stratified Randomized Field Trial. J Clin Endocrinol Metab 2018; 103: 2936-48.

27. Curtis EM, Moon RJ, Harvey NC, Cooper C. Maternal vitamin D supplementation during pregnancy. Br Med Bull 2018; 126: 5777.

28. Medicine I, Board FN, Calcium CRDRIVD, Valle HBD, Yaktine AL, Taylor CL, et al. Institute of Medicine, Food and Nutrition Board, Committee to Review Dietary Reference Intakes for Vitamin D and Calcium. In: Del Valle HB, Yaktine AL, Taylor CL, Catharine Ross A, editors. Dietary Reference Intakes for Calcium and Vitamin D. National Academies Press (US); 2011.

29. Chauhan K, Shahrokhi M, Huecker MR. Vitamin D. 2021 Jul 11. In: StatPearls [Internet]. Treasure Island (FL): StatPearls Publishing; 2021. Available from: http://www.ncbi.nlm.nih.gov/ pubmed/28722941

30. Knabl J, Vattai A, Ye Y, Jueckstock J, Hutter S, Kainer F, et al. Role of Placental VDR Expression and Function in Common Late Pregnancy Disorders. Int J Mol Sci 2017; 18: 2340.

31. Jones KS, Assar S, Harnpanich D, Bouillon R, Lambrechts D, Prentice A, et al. 25(OH)D2 half-life is shorter than 25(OH)D3 halflife and is influenced by DBP concentration and genotype. J Clin Endocrinol Metab 2014; 99: 3373-81.

32. Hollis BW, Wagner CL. Clinical review: Clinical review: The role of the parent compound vitamin D with respect to metabolism and 
function: Why clinical dose intervals can affect clinical outcomes. $\mathrm{J}$ Clin Endocrinol Metab 2013; 98: 4619-28.

33. Yazdchi R, Gargari BP, Asghari-Jafarabadi M, Sahhaf F. Effects of vitamin $\mathrm{D}$ supplementation on metabolic indices and hs-CRP levels in gestational diabetes mellitus patients: a randomized, doubleblinded, placebo-controlled clinical trial. Nutr Res Pract 2016; 10: 328-35.

34. Asemi Z, Karamali M, Esmaillzadeh A. Effects of calcium-vitamin D co-supplementation on glycaemic control, inflammation and oxidative stress in gestational diabetes: a randomised placebocontrolled trial. Diabetologia 2014; 57: 1798-806.

35. Jamilian M, Samimi M, Ebrahimi FA, Hashemi T, Taghizadeh M, Razavi M, et al. The effects of vitamin D and omega-3 fatty acid cosupplementation on glycemic control and lipid concentrations in patients with gestational diabetes. J Clin Lipidol 2017; 11: 459-68.

36. Asemi Z, Hashemi T, Karamali M, Samimi M, Esmaillzadeh A. Effects of vitamin D supplementation on glucose metabolism, lipid concentrations, inflammation, and oxidative stress in gestational diabetes: a double-blind randomized controlled clinical trial. Am J Clin Nutr 2013; 98: 1425-32.

37. Li Q, Xing B. Vitamin D3-Supplemented Yogurt Drink Improves Insulin Resistance and Lipid Profiles in Women with Gestational Diabetes Mellitus: A Randomized Double Blinded Clinical Trial. Ann Nutr Metab 2016; 68: 285-90.

38. Hosseinzadeh-Shamsi-Anar M, Mozaffari-Khosravi H, Salami MA, Hadinedoushan $\mathrm{H}$, Mozayan MR. The efficacy and safety of a high dose of vitamin $\mathrm{d}$ in mothers with gestational diabetes mellitus: a randomized controlled clinical trial. Iran J Med Sci 2012; 37: 15965.

39. Saha S, Saha S. The risk of morbidities in newborns of antenatal vitamin D supplemented gestational diabetes mellitus patient. Int $\mathrm{J}$ Health Sci 2020; 14: 3-17.

40. Saha S, Saha S. A comparison of the risk of cesarean section in gestational diabetes mellitus patients supplemented antenatally with vitamin D containing supplements versus placebo: A systematic review and meta-analysis of double-blinded randomized controlled trials. J Turk Ger Gynecol Assoc 2020; 21: 201-12.

41. Saha S. Obstetric and neonatal outcomes in vitamin D supplemented gestational diabetes mellitus patients: an abridgment of systematic reviews. AIMS Med Sci 2020; 7: 298-300.

42. Saha S, Saha S. A Comparison of the Changes in Gestational Weight, Body Mass Index, and Serum Vitamin D Level in Gestational Diabetes Mellitus Patients Complemented with Vitamin D in Contrast to Those Who Did Not Receive the Supplement: A Protocol for Systematic Review and Meta-Analysis of Randomised Controlled Trials. Int J Diabetes Metab 2019; 25: 74-9.

43. Saha S, Saha S. A comparison of changes in gestational weight, body mass index and serum vitamin $\mathrm{D}$ level in gestational diabetes mellitus patients complemented with vitamin D contrasted to those who did not receive the supplement: a protocol for systematic review and meta-analysis of randomised controlled trials. PROSPERO. 2020 [cited: 2020 Apr 11]. p. CRD42020149613. Available from: https://www.crd.york.ac.uk/prospero/display_ record.php?ID $=$ CRD42020149613

44. Liberati A, Altman DG, Tetzlaff J, Mulrow C, Gøtzsche PC, Ioannidis JPA, et al. The PRISMA statement for reporting systematic reviews and meta-analyses of studies that evaluate health care interventions: explanation and elaboration. J Clin Epidemiol 2009; 62: e1-34.
45. Ouzzani M, Hammady H, Fedorowicz Z, Elmagarmid A. Rayyan-a web and mobile app for systematic reviews. Syst Rev 2016; 5: 210.

46. Higgins JPT GS (editors). Cochrane Handbook for Systematic Reviews of Interventions Version 5.1.0 [updated March 2011]. [Internet]. The Cochrane Collaboration. 2011 [cited: 2020 Aug 27]. Available from: https://training.cochrane.org/handbook/archive/ v5.1/

47. The Cochrane Collaboration. Review Manager (RevMan) [Computer program] [Internet]. The Cochrane Collaboration. Copenhagen: The Nordic Cochrane Centre; 2014. Available from: https://training. cochrane.org/online-learning/core-software-cochrane-reviews/ revman/revman-5-download/download-and-installation

48. Higgins JP, Thompson SG, Deeks JJ, Altman DG. Measuring inconsistency in meta-analyses. BMJ 2003; 327: 557-60.

49. Asemi Z, Karamali M, Esmaillzadeh A. Favorable effects of vitamin D supplementation on pregnancy outcomes in gestational diabetes: a double blind randomized controlled clinical trial. Horm Metab Res 2015; 47: 565-70.

50. Jamilian M, Amirani E, Asemi Z. The effects of vitamin D and probiotic co-supplementation on glucose homeostasis, inflammation, oxidative stress and pregnancy outcomes in gestational diabetes: A randomized, double-blind, placebo-controlled trial. Clin Nutr 2019; 38: 2098-105.

51. Razavi M, Jamilian M, Samimi M, Afshar Ebrahimi F, Taghizadeh M, Bekhradi R, et al. he effects of vitamin D and omega-3 fatty acids cosupplementation on biomarkers of inflammation, oxidative stress and pregnancy outcomes in patients with gestational diabetes. Nutr Metab 2017; 14: 80.

52. Rossi G; American Diabetes Association. Diagnosis and classification of diabetes mellitus. Recenti Prog Med 2010; 101: 2746.

53. American Diabetes Association. Diagnosis and classification of diabetes mellitus. Diabetes Care 2014; 37Suppl1: S81-90.

54. Atkins D, Best D, Briss PA, Eccles M, Falck-Ytter Y, Flottorp S, et al. Grading quality of evidence and strength of recommendations. BMJ 2004; 328: 1490.

55. Mallet E, Gügi B, Brunelle P, Hénocq A, Basuyau JP, Lemeur H. Vitamin D supplementation in pregnancy: a controlled trial of two methods. Obstet Gynecol 1986; 68: 300-4.

56. Yu CK, Sykes L, Sethi M, Teoh TG, Robinson S. Vitamin D deficiency and supplementation during pregnancy. Clin Endocrinol 2009; 70: 685-90.

57. Roth DE, Al Mahmud A, Raqib R, Akhtar E, Perumal N, Pezzack B, et al. andomized placebo-controlled trial of high-dose prenatal thirdtrimester vitamin D3 supplementation in Bangladesh: the AViDD trial. Nutr J 2013; 12: 47.

58. Moher D, Liberati A, Tetzlaff J, Altman DG; PRISMA Group. Preferred reporting items for systematic reviews and meta-analyses: the PRISMA statement. PLoS Med 2009; 6: e1000097.

59. Asemi Z. Clinical trial of the effect of combined omega-3 fatty acids and vitamin D supplementation compared with the placebo on metabolic profiles and pregnancy outcomes in patients with gestational diabetes (Internet). Iranian Registry of Clinical Trials. 2017 (cited: 2019 Oct 18). p. Clinical Trial Protocol. Available from: http://en.irct.ir/trial/6136

60. Clinical trial of the effect of combined vitamin D and evening primrose oil supplementation on metabolic profiles in gestational diabetes (Internet). IRCT (cited: 2020 Jun 9). Available from: https:// www.irct.ir/trial/6082 
Supplement Table 1. PRISMA checklist

\begin{tabular}{|c|c|c|c|}
\hline Section/topic & \# & Checklist item & $\begin{array}{l}\text { Reported on } \\
\text { page \# }\end{array}$ \\
\hline Title & 1 & Identify the report as a systematic review, meta-analysis, or both. & 1 \\
\hline \multicolumn{4}{|l|}{ Abstract } \\
\hline Structured summary & 2 & $\begin{array}{l}\text { Provide a structured summary including, as applicable: background; objectives; data sources; study } \\
\text { eligibility criteria, participants, and interventions; study appraisal and synthesis methods; results; } \\
\text { limitations; conclusions and implications of key findings; systematic review registration number. }\end{array}$ & $2-3$ \\
\hline \multicolumn{4}{|l|}{ Introduction } \\
\hline Rationale & 3 & Describe the rationale for the review in the context of what is already known. & $4-6$ \\
\hline Objectives & 4 & $\begin{array}{l}\text { Provide an explicit statement of questions being addressed with reference to participants, } \\
\text { interventions, comparisons, outcomes, and study design (PICOS). }\end{array}$ & 6 \\
\hline \multicolumn{4}{|l|}{ Methods } \\
\hline $\begin{array}{l}\text { Protocol and } \\
\text { registration }\end{array}$ & 5 & $\begin{array}{l}\text { Indicate if a review protocol exists, if and where it can be accessed (e.g., Web address), and, if } \\
\text { available, provide registration information including registration number. }\end{array}$ & 6 \\
\hline Eligibility criteria & 6 & $\begin{array}{l}\text { Specify study characteristics (e.g., PICOS, length of follow-up) and report characteristics (e.g., years } \\
\text { considered, language, publication status) used as criteria for eligibility, giving rationale. }\end{array}$ & 7 \\
\hline Information sources & 7 & $\begin{array}{l}\text { Describe all information sources (e.g., databases with dates of coverage, contact with study authors } \\
\text { to identify additional studies) in the search and date last searched. }\end{array}$ & 7 \\
\hline Search & 8 & $\begin{array}{l}\text { Present full electronic search strategy for at least one database, including any limits used, such that } \\
\text { it could be repeated. }\end{array}$ & $7-8$ \\
\hline Study selection & 9 & $\begin{array}{l}\text { State the process for selecting studies (i.e., screening, eligibility, included in systematic review, and, } \\
\text { if applicable, included in the meta-analysis). }\end{array}$ & 8 \\
\hline $\begin{array}{l}\text { Data collection } \\
\text { process }\end{array}$ & 10 & $\begin{array}{l}\text { Describe method of data extraction from reports (e.g., piloted forms, independently, in duplicate) } \\
\text { and any processes for obtaining and confirming data from investigators. }\end{array}$ & 8 \\
\hline Data items & 11 & $\begin{array}{l}\text { List and define all variables for which data were sought (e.g., PICOS, funding sources) and any } \\
\text { assumptions and simplifications made. }\end{array}$ & 8 \\
\hline $\begin{array}{l}\text { Risk of bias in } \\
\text { individual studies }\end{array}$ & 12 & $\begin{array}{l}\text { Describe methods used for assessing risk of bias of individual studies (including specification of } \\
\text { whether this was done at the study or outcome level), and how this information is to be used in any } \\
\text { data synthesis. }\end{array}$ & $8-9$ \\
\hline Summary measures & 13 & State the principal summary measures (e.g., risk ratio, difference in means). & 9 \\
\hline Synthesis of results & 14 & $\begin{array}{l}\text { Describe the methods of handling data and combining results of studies, if done, including } \\
\text { measures of consistency (e.g., } I^{2} \text { ) for each meta-analysis. }\end{array}$ & 9 \\
\hline $\begin{array}{l}\text { Risk of bias across } \\
\text { studies }\end{array}$ & 15 & $\begin{array}{l}\text { Specify any assessment of risk of bias that may affect the cumulative evidence (e.g., publication } \\
\text { bias, selective reporting within studies). }\end{array}$ & 10 \\
\hline Additional analyses & 16 & $\begin{array}{l}\text { Describe methods of additional analyses (e.g., sensitivity or subgroup analyses, meta-regression), if } \\
\text { done, indicating which were pre-specified. }\end{array}$ & 10 \\
\hline \multicolumn{4}{|l|}{ Results } \\
\hline Study selection & 17 & $\begin{array}{l}\text { Give numbers of studies screened, assessed for eligibility, and included in the review, with reasons } \\
\text { for exclusions at each stage, ideally with a flow diagram. }\end{array}$ & 10 \\
\hline Study characteristics & 18 & $\begin{array}{l}\text { For each study, present characteristics for which data were extracted (e.g., study size, PICOS, } \\
\text { follow-up period) and provide the citations. }\end{array}$ & $10-11$ \\
\hline $\begin{array}{l}\text { Risk of bias within } \\
\text { studies }\end{array}$ & 19 & $\begin{array}{l}\text { Present data on risk of bias of each study and, if available, any outcome level assessment (see item } \\
\text { 12). }\end{array}$ & 11 \\
\hline $\begin{array}{l}\text { Results of individual } \\
\text { studies }\end{array}$ & 20 & $\begin{array}{l}\text { For all outcomes considered (benefits or harms), present, for each study: (a) simple summary data } \\
\text { for each intervention group (b) effect estimates and confidence intervals, ideally with a forest plot. }\end{array}$ & 12 \\
\hline Synthesis of results & 21 & $\begin{array}{l}\text { Present results of each meta-analysis done, including confidence intervals and measures of } \\
\text { consistency. }\end{array}$ & $11-12$ \\
\hline $\begin{array}{l}\text { Risk of bias across } \\
\text { studies }\end{array}$ & 22 & Present results of any assessment of risk of bias across studies (see Item 15). & $11-12$ \\
\hline
\end{tabular}


Supplement Table 1. Continued

\begin{tabular}{|c|c|c|c|}
\hline Section/topic & $\#$ & Checklist item & $\begin{array}{l}\text { Reported on } \\
\text { page \# }\end{array}$ \\
\hline \multicolumn{4}{|l|}{ Results } \\
\hline Additional analysis & 23 & $\begin{array}{l}\text { Give results of additional analyses, if done (e.g., sensitivity or subgroup analyses, meta- } \\
\text { regression [see Item 16]). }\end{array}$ & $11-12$ \\
\hline \multicolumn{4}{|l|}{ Discussion } \\
\hline Summary of evidence & 24 & $\begin{array}{l}\text { Summarize the main findings including the strength of evidence for each main outcome; } \\
\text { consider their relevance to key groups (e.g., healthcare providers, users, and policy makers). }\end{array}$ & 13 \\
\hline Limitations & 25 & $\begin{array}{l}\text { Discuss limitations at study and outcome level (e.g., risk of bias), and at review-level (e.g., } \\
\text { incomplete retrieval of identified research, reporting bias). }\end{array}$ & 14 \\
\hline Conclusions & 26 & $\begin{array}{l}\text { Provide a general interpretation of the results in the context of other evidence, and implications } \\
\text { for future research. }\end{array}$ & 14 \\
\hline \multicolumn{4}{|l|}{ Funding } \\
\hline Funding & 27 & $\begin{array}{l}\text { Describe sources of funding for the systematic review and other support (e.g., supply of data); } \\
\text { role of funders for the systematic review. }\end{array}$ & 14 \\
\hline
\end{tabular}

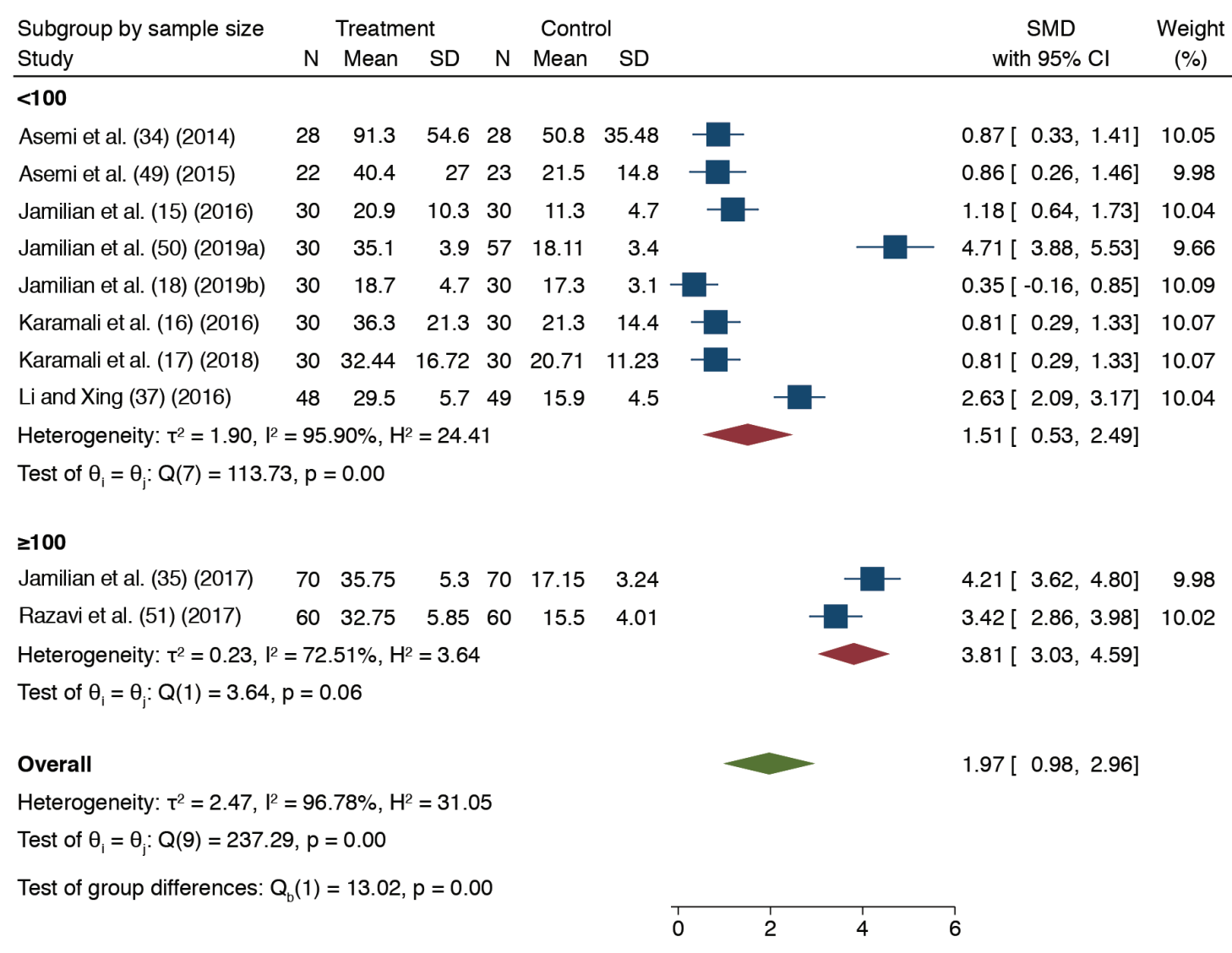

Supplement Figure 1. Forest plot showing meta-analysis comparing between antenatal vitamin D supplementation (as a sole or co-supplement with other supplements) and non-vitamin D supplementation results on 25(OH)D level in blood using random-effect model. Subgroup by sample size $(<100$ and $\geq 100$ category); Two trials have identical trial author name and year that have been suffixed with alphabet " $a$ " (61) and "b" (27) after the study year

SD: Standard deviation, CI: Confidence interval, SMD: Standardized mean difference, 25(OH)D: 25-hydroxyvitamin D 


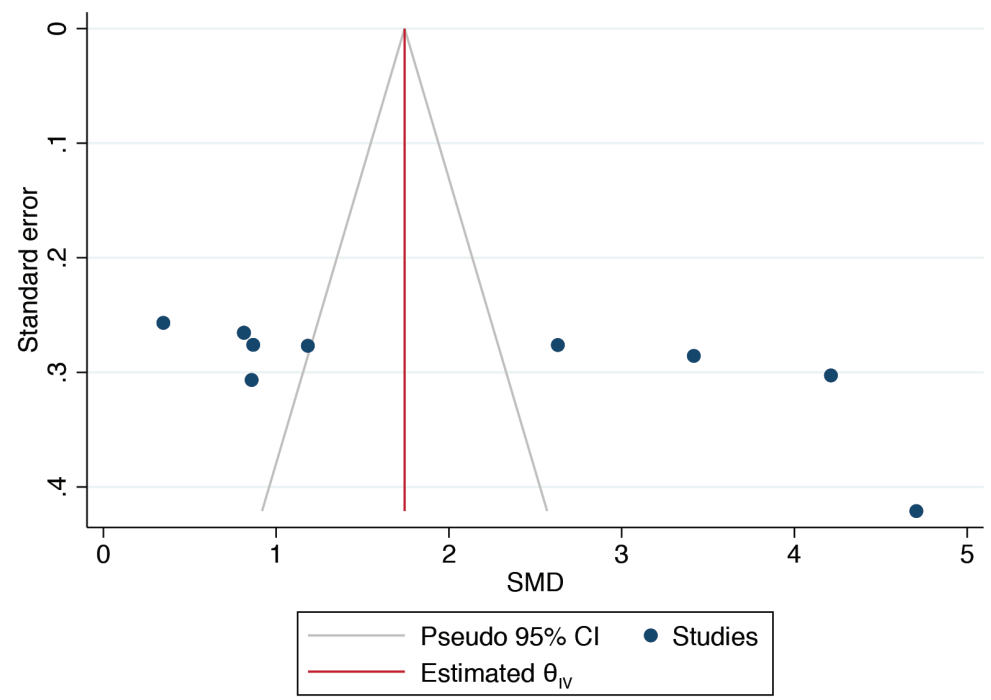

Supplement Figure 2. Funnel plot assessing publication bias between vitamin D supplemented and not supplemented GDM mothers for 25(OH)D levels in the blood

GDM: Gestational diabetes mellitus, SMD: Standardized mean difference, CI: Confidence interval, 25(OH)D: 25-hydroxyvitamin D

Supplement Table 2. Univariate meta-regression analysis

\begin{tabular}{|c|c|c|c|c|}
\hline \multirow{2}{*}{\multicolumn{2}{|c|}{ Category }} & \multicolumn{3}{|c|}{ Univariate model } \\
\hline & & Estimate & p-value & 95\% CI \\
\hline Participant attrition & No & 1 & - & - \\
\hline \multirow[t]{2}{*}{ Sample size } & $<100$ & 1 & - & - \\
\hline & $\geq 100$ & 2.31 & $0.028^{*}$ & $0.25,4.37$ \\
\hline
\end{tabular}

